\title{
Optical and spectral observations and hydrodynamic modelling of Type IIb Supernova 2017gpn
}

\author{
Elena A. Balakina, ${ }^{1,2}$ Maria V. Pruzhinskaya ${ }^{2,3 \star}$ Alexander S. Moskvitin, ${ }^{4}$ \\ Sergei I. Blinnikov, ${ }^{2,3,5,6}$ Xiaofeng Wang, ${ }^{7,8}$ Danfeng Xiang, ${ }^{7}$ Han Lin, ${ }^{7}$ \\ Liming Rui ${ }^{7}$, and Huijuan Wang ${ }^{9}$ \\ ${ }^{1}$ Lomonosov Moscow State University, Faculty of Physics, Leninskie Gory, 1-2, Moscow, 119991, Russia \\ ${ }^{2}$ Lomonosov Moscow State University, Sternberg Astronomical Institute, Universitetsky pr. 13, Moscow, 119234, Russia \\ 3 Space Research Institute, 84/32 Profsoyuznaya Street, Moscow, 117997, Russia \\ ${ }^{4}$ Special Astrophysical Observatory RAS, Nizhnij Arhyz, 369167, Russia \\ ${ }^{5}$ NRC Kurchatov institute - ITEP, B.Cheremushkinskaya 25, 117218 Moscow, Russia \\ ${ }^{6}$ Kavli IPMU, University of Tokyo, Kashiwa, 277-8583, Japan \\ ${ }^{7}$ Physics Department and Tsinghua Center for Astrophysics, Tsinghua Unviersity, Beijing, 100084, China \\ ${ }^{8}$ Beijing Planetarium, Beijing Academy of Science and Technology, Beijing, 100044, China \\ ${ }^{9}$ National Astronomical Observatories, Chinese Academy of Sciences, Beijing, 100101, China
}

Accepted XXX. Received YYY; in original form ZZZ

\begin{abstract}
In this work we present the photometric and spectroscopic observations of Type IIb Supernova 2017gpn. This supernova was discovered in the error-box of the LIGO/Virgo G299232 gravitational-wave event. We obtained the light curves in the $B$ and $R$ passbands and modelled them numerically using the one-dimensional radiation hydrocode STELLA. The best-fitting model has the following parameters: the pre-SN star mass and the radius are $\mathrm{M} \approx 3.5 M_{\odot}$ and $\mathrm{R} \approx 50 R_{\odot}$, respectively; the explosion energy is $\mathrm{E}_{\exp } \approx 1.2 \times 10^{51} \mathrm{erg}$; the mass of radioactive nickel is $\mathrm{M}_{56 \mathrm{Ni}} \approx 0.11 M_{\odot}$, which is completely mixed throughout the ejecta; and the mass of the hydrogen envelope $\mathrm{M}_{\mathrm{H} \_ \text {env }} \approx 0.06 M_{\odot}$. Moreover, $\mathrm{SN} 2017 \mathrm{gpn}$ is a confirmed SN IIb that is located at the farthest distance from the centre of its host galaxy NGC 1343 (i.e. the projected distance is $\sim 21 \mathrm{kpc}$ ). This challenges the scenario of the origin of Type IIb Supernovae from massive stars.
\end{abstract}

Key words: supernovae: general - supernovae: individual: SN 2017gpn - stars: evolution

\section{INTRODUCTION}

Type IIb Supernovae (SNe IIb) are characterized by spectra evolving from dominant hydrogen lines at early times to increasingly strong helium features and progressively weaker hydrogen lines later on (Filippenko et al. 1993). This is the reason why SNe IIb are regarded as an intermediate group between hydrogen-rich $\mathrm{SNe}$ II and hydrogen-poor $\mathrm{SNe} \mathrm{Ib}$. SNe IIb are in the class of the strippedenvelope core-collapse supernovae (CCSNe). It is supposed that progenitors of such supernovae are massive stars that have lost most of their hydrogen envelope (Clocchiatti \& Wheeler 1997).

Nowadays there are two hypotheses explaining how stars can lose the hydrogen envelope. The first scenario supposes the evolution of a rather massive $\mathrm{M} \simeq 25 M_{\odot}$ single star with an average mass-loss rate of about $10^{-5} M_{\odot}$ per year. Such a powerful stellar wind could provide the required outflow of hydrogen (Hoflich et al.
1993). The second and more plausible scenario involves a mass transfer in a binary system where the progenitor star is a supergiant of moderate mass (Nomoto et al. 1993; Woosley et al. 1994; Bersten et al. 2012). The massive companion expands and fills its Roche lobe, after which mass transfer starts due to Roche-lobe overflow (Yoon et al. 2017).

Nevertheless, the progenitor nature of SNe IIb is still not clear. While SNe II form a continuous group as Anderson et al. (2014) and Sanders et al. (2015) established, Pessi et al. (2019) showed that SN II light curves are distinct from those of SNe IIb with no suggestion of a continuum distribution. This fact suggests that progenitors of $\mathrm{SNe}$ IIb make up a separate group that is different from the SNe II ones. However, it could also be a consequence of the lack of observational data: SNe IIb make up less than 5 per cent of all CC$\mathrm{SNe}$ according to the Open Supernova Catalog ${ }^{1}$ (Guillochon et al. 2017) and only about two dozen of them have detailed multicolour 
photometry appropriate for further study (including hydrodynamic modelling).

To extend the sample of well-studied $\mathrm{SNe} \mathrm{IIb}$, in this paper we present the photometric and spectroscopic observations of SN 2017gpn. The photometry was performed with the Zeiss-1000 telescope (Komarov et al. 2020) at the Special Astrophysical Observatory of the Russian Academy of Science (SAO RAS). Spectroscopic data were obtained with the Xinglong 2.16-m telescope at the National Astronomical Observatory of China. Collected photometric data are used for the numerical light-curve (LC) calculations done by the radiation hydrocode STELLA (Blinnikov et al. 1998, 2006). These simulations give us the parameters of the presupernova star and explosion characteristics.

The interest in this supernova is also augmented by the fact that we usually only observe such supernovae in spiral galaxies in hydrogen-rich environments where young massive stars are being born (Filippenko 1997). In contrast to this, SN 2017gpn is located quite far from the active star-formation regions and the spiral arms of the host galaxy. We also do not see any dwarf satellite galaxies at the SN location. The unusual location of SN 2017gpn in the host galaxy indicates that the existing models of SN IIb progenitors may not explain all observational data and have to be reviewed.

This paper is organized as follows. In Section 2 we describe the observations, data processing, and resulting light curves and spectra. In Section 3 we present the hydrodynamic modelling of SN 2017gpn and the parameters of the best-fitting model. Section 4 contains a comparison of the modelling results, LC behaviour, and spectral features of SN 2017gpn with those for other SNe IIb and a discussion of the unexpected location of SN 2017gpn relative to its host galaxy. Finally, we conclude the paper in Section 5.

\section{OBSERVATIONS}

\subsection{Discovery}

On the last day of the second advanced detector observing run "O2", the LIGO/Virgo Collaboration released the G299232 alert $^{2}$. During the follow-up inspection of the gravitational-wave $(\mathrm{GW})$ candidate error-box, on 2017 August 27.017 the MASTER Global Robotic Net (Lipunov et al. 2010) discovered an optical transient named MASTER OT J033744.97+723159.0 (Lipunov et al. 2017).

On the discovery day, three spectra of MASTER OT J033744.97+723159.0 were obtained with the ACAM instrument mounted on the William Herschel Telescope at La Palma (Spain) by Jonker et al. (2017) and the analysis showed that the transient classifies as SNe IIb. Further observations on $2017 \mathrm{Au}-$ gust 29 obtained with the SPRAT spectrograph on the Liverpool Telescope (Copperwheat et al. 2017) and with the Xinglong 2.16-m telescope of the National Astronomical Observatory of China (Rui et al. 2017; Wang 2017) confirmed this classification by crosscorrelating with a library of spectra with use of the Supernova Identification code (SNID; Blondin \& Tonry 2007). According to SNID, the spectrum with the highest correlation coefficient belongs to Type IIb SN $1996 \mathrm{cb}$ at phase $-2 \mathrm{~d}$.

On 2017 September 6 at 03:21:12 UT, Caimmi (2017) reported the discovery of a supernova with the 0.24-m telescope from the Valdicerro Observatory. The supernova received the IAU designation AT $2017 \mathrm{gpn}$ and was identified as MASTER OT J033744.97+723159.0.

\footnotetext{
2 https://gcn.gsfc.nasa.gov/other/G299232.gcn3
}

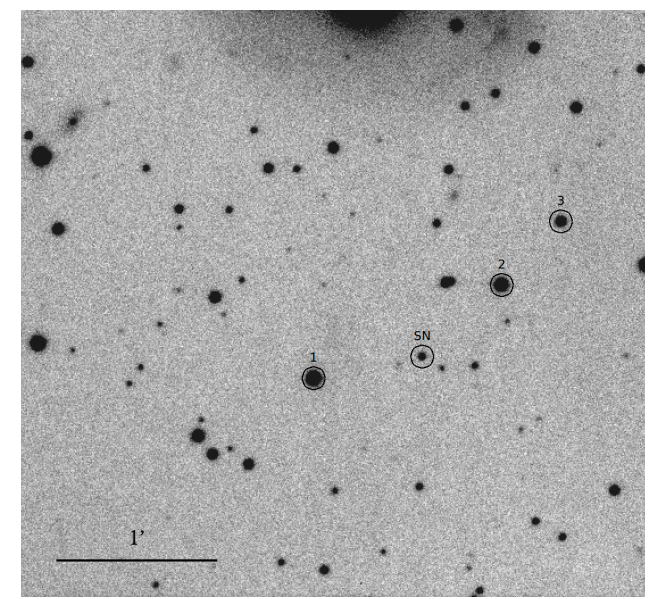

Figure 1. SN 2017gpn and comparison stars. The image is obtained with the Zeiss-1000 telescope in $R$ passband.

Table 1. Magnitudes of the comparison stars in the $B$ and $R$ passbands derived from $g, r, i$ Pan-STARRS1 magnitudes using Lupton's 2005 transformation equations.

\begin{tabular}{ccccc}
\hline № & $B$ & $\operatorname{err}_{B}$ & $R$ & $\operatorname{err}_{R}$ \\
\hline 1 & 16.447 & 0.011 & 15.032 & 0.015 \\
2 & 16.859 & 0.012 & 15.428 & 0.015 \\
3 & 17.705 & 0.011 & 16.636 & 0.017 \\
\hline
\end{tabular}

SN 2017gpn is located $\sim 140$ arcsec from the centre of the host galaxy NGC 1343 (Fig. 1). Taking into account that the redshift of NGC 1343 is 0.0073 (Springob et al. 2005) and assuming flat $\Lambda \mathrm{CDM}$ cosmology with $\Omega_{\Lambda}=0.7$ and $H_{0}=70 \mathrm{~km} \mathrm{~s}^{-1} \mathrm{Mpc}^{-1}$, we find that the projected distance between SN 2017gpn and the centre of its host is $\sim 21 \mathrm{kpc}$.

\subsection{Photometric data processing}

We performed 20 epochs of observations ( $B$ and $R$ passbands) with the CCD photometer on the Zeiss-1000 telescope at SAO RAS. The aperture photometry was performed using standard procedures of the ESO MIDAS software package. It includes standard image processing such as bias subtraction and flat field correction, removing the traces of cosmic particles, and stacking of individual frames into a summary image.

Since no Landolt or any other standards (Stetson 1987; Landolt 1992) were available for this region, we use the PanSTARRS (Chambers et al. 2016; Flewelling et al. 2016) magnitudes for comparison stars. These magnitudes were recalculated from the $g, r, i$ passbands to $B$ and $R$ with the use of Lupton's transformation equations $^{3}$ :

$$
\begin{array}{lll}
B=g+0.3130(g-r)+0.2271, & \sigma=0.0107 \\
R=r-0.1837(g-r)-0.0971, & \sigma=0.0106 \\
R=r-0.2936(r-i)-0.1439, & \sigma=0.0072
\end{array}
$$

3 http://www.sdss3.org/dr8/algorithms/ sdssUBVRITransform.php 
Table 2. Photometric observations of SN 2017gpn with the Zeiss-1000 telescope. The magnitudes are corrected for the expected Galactic foreground extinction.

\begin{tabular}{lcccc}
\hline JD 2457990+ & $B$ & $\operatorname{err}_{B}$ & $R$ & $\operatorname{err}_{R}$ \\
\hline 21.5 & 16.65 & 0.07 & 15.34 & 0.03 \\
22.5 & 16.75 & 0.05 & 15.41 & 0.02 \\
25.6 & 17.08 & 0.05 & 15.58 & 0.03 \\
26.5 & 17.16 & 0.05 & 15.62 & 0.04 \\
27.5 & 17.24 & 0.06 & 15.67 & 0.02 \\
28.5 & 17.33 & 0.06 & 15.73 & 0.02 \\
29.6 & 17.35 & 0.05 & 15.81 & 0.02 \\
31.5 & 17.44 & 0.06 & 15.88 & 0.03 \\
56.4 & 17.90 & 0.06 & 16.62 & 0.02 \\
57.4 & 17.89 & 0.05 & 16.63 & 0.01 \\
76.5 & - & - & 17.17 & 0.03 \\
77.4 & 18.17 & 0.07 & 17.13 & 0.03 \\
78.6 & 18.14 & 0.06 & 17.21 & 0.03 \\
85.6 & 18.22 & 0.05 & 17.21 & 0.03 \\
107.6 & - & - & 18.06 & 0.04 \\
110.4 & 18.61 & 0.07 & 17.89 & 0.03 \\
143.3 & 19.14 & 0.15 & 18.78 & 0.01 \\
153.3 & - & - & 18.54 & 0.30 \\
224.3 & - & - & 21.14 & 0.20 \\
\hline & & & &
\end{tabular}

The comparison stars are shown in Fig. 1 and their magnitudes are listed in Table 1.

We use a line-of-sight reddening for our Galaxy of $E(B-V)=$ 0.30 mag (Schlafly \& Finkbeiner 2011), corresponding to additive magnitude corrections of 1.246 and $0.725 \mathrm{mag}$ for the $B$ and $R$ passbands, respectively. Since SN 2017gpn is very far from the centre of NGC 1343, we assume that the host's contamination is negligible. The resulting photometric data are presented in Table 2.

\subsection{Resulting light curves}

With Zeiss-1000 observations we can restore only the postmaximum part of the light curve. This is why, to improve the accuracy of the further hydrodynamic modelling (see Section 3), we supplemented our data with observations in the $B$ and $R$ passbands from Roberts \& Kolb 2018 obtained with the PIRATE robotic telescope in Spain (Holmes et al. 2011). The resulting light curve is presented in Fig. 2. The data points obtained at Zeiss-1000 (shown as circles) and the data points taken from Roberts \& Kolb 2018 (marked with crosses) mutually complement each other and allow us to restore the $B$ and $R$ light curves almost entirely.

One can notice a slight shift between the two data sets. This may be due to the different sources of photometry for the comparison stars since there are no Stetson and Landolt photometric standards in this field. However, the difference between the values is less than the uncertainty associated with the choice of hydrodynamic model; therefore for our purpose it can be neglected.

\subsection{Spectra}

The spectroscopic observations were collected using the Xinglong 2.16-m telescope and the BFOSC system. All the spectra were reduced using routine tasks within IRAF and the flux was calibrated with spectrophotometric standard stars observed on the same nights. Telluric lines are removed from all of these spectra. The journal of our spectroscopic observations is given in Table 3.

Three optical spectra were obtained for SN $2017 \mathrm{gpn}$, covering

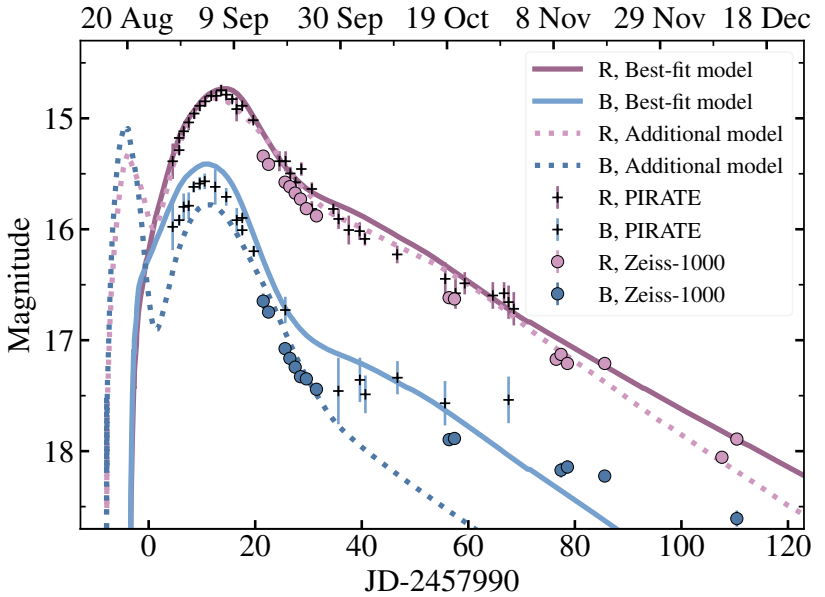

Figure 2. Light curve of SN 2017gpn. Pink and blue solid lines correspond to the best-fitting model; dashed lines to the additional model in the $R$ and $B$ passbands, respectively. Circles are the Zeiss-1000 data; crosses are the data taken from Roberts \& Kolb 2018.

the phases from -8.3 to $+19.7 \mathrm{~d}$ from the $R$-band maximum light (peak time is JD = 2458003.6); these are shown in Fig. 3. At one week before the peak, the spectrum shows strong Balmer lines of hydrogen, providing evidence of a Type II Supernova. Moreover, the existing prominent absorption features at $\sim 5670$ and $6860 \AA \AA$ that can be identified as $\mathrm{He}_{\text {I }} \lambda 5876$ and $\mathrm{He}_{\text {I }} \lambda 7065$, respectively, confirming that SN 2017gpn can be further put into the Type IIb subclass. From the absorption minima of $\mathrm{H} \alpha$ and $\mathrm{He}_{\mathrm{I}} \lambda 5876$ lines at the first obtained spectrum, we measured the ejecta velocity as $15000 \pm 130$ and $10100 \pm 300 \mathrm{~km} \mathrm{~s}^{-1}$, respectively, indicating that the Balmer lines and the $\mathrm{He}_{\mathrm{I}}$ lines originated from different layers (see Table 3). At two weeks after the maximum, the helium features seem to become more noticeable and other helium features such as $\mathrm{He}_{\mathrm{I}} \lambda 6678$ (blueshifted to $\sim 6510 \AA$ ) emerge in the spectrum. The helium features become even more pronounced in the spectrum taken one week later, while the hydrogen features become gradually weaker. The overall spectral evolution of SN 2017gpn is presented in Fig. 3 and it is similar to other typical Type IIb Supernovae, like SN 1993J (Barbon et al. 1995), SN 1996cb (Qiu et al. 1999), and SN 2008ax (Modjaz et al. 2014).

\section{MODELLING}

\subsection{Pre-supernova models}

A set of non-evolutionary pre-supernova models is obtained under the assumption of a power-law dependence of temperature on density: $T \propto \rho^{\alpha}$ (Nadyozhin \& Razinkova 1986; Blinnikov \& Bartunov 1993). Therefore, the obtained hydrostatic configuration would be close to a polytrope of index $1 / \alpha \simeq 3$. The deviation from the polytropic model increases in the outer layers due to recombination of ions and non-homogeneous chemical composition.

At the centre of such a configuration we isolated a point-like source of gravity that has a non-negligible influence on the expansion of the innermost layers of supernova ejecta. The mass and radius of this compact remnant are taken as $\mathrm{M}_{\mathrm{CR}}=1.41 M_{\odot}$ and $0.01 R_{\odot}$ for all treated pre-SN models.

In our approach we do not follow the explosive nucleosynthesis. Thus, the SN ejecta composition is the same as the pre-SN 
Table 3. Journal of spectroscopic observations of SN 2017gpn with the BFOSC+G4 instrument of the Xinglong 2.16-m telescope. Values of the ejecta velocity measured from the absorption lines of $\mathrm{H} \alpha$, He I $\lambda 2876$, Fe II $\lambda 5018$, and Fe II $\lambda 5169$ are also presented.

\begin{tabular}{lccccc}
\hline JD 2457990+ & $\begin{array}{c}\text { Exp. Time } \\
{[\mathrm{s}]}\end{array}$ & $\begin{array}{c}\mathrm{H} \alpha \\
{\left[\mathrm{km} \mathrm{s}^{-1}\right]}\end{array}$ & $\begin{array}{c}\mathrm{He}_{\mathrm{I}} \lambda 5876 \\
{\left[\mathrm{~km} \mathrm{~s}^{-1}\right]}\end{array}$ & $\begin{array}{c}\text { Fe II } \lambda 5018 \\
{\left[\mathrm{~km} \mathrm{~s}^{-1}\right]}\end{array}$ & $\begin{array}{c}\text { Fe II } \lambda 5169 \\
{\left[\mathrm{~km} \mathrm{~s}^{-1}\right]}\end{array}$ \\
\hline 5.30 & 3600 & $15000 \pm 130$ & $10100 \pm 300$ & $12000 \pm 1200$ & $11400 \pm 950$ \\
25.29 & 3600 & $13200 \pm 100$ & $8000 \pm 100$ & $6750 \pm 470$ & $5130 \pm 490$ \\
33.33 & 2700 & $12900 \pm 200$ & $7300 \pm 200$ & - & - \\
\hline
\end{tabular}

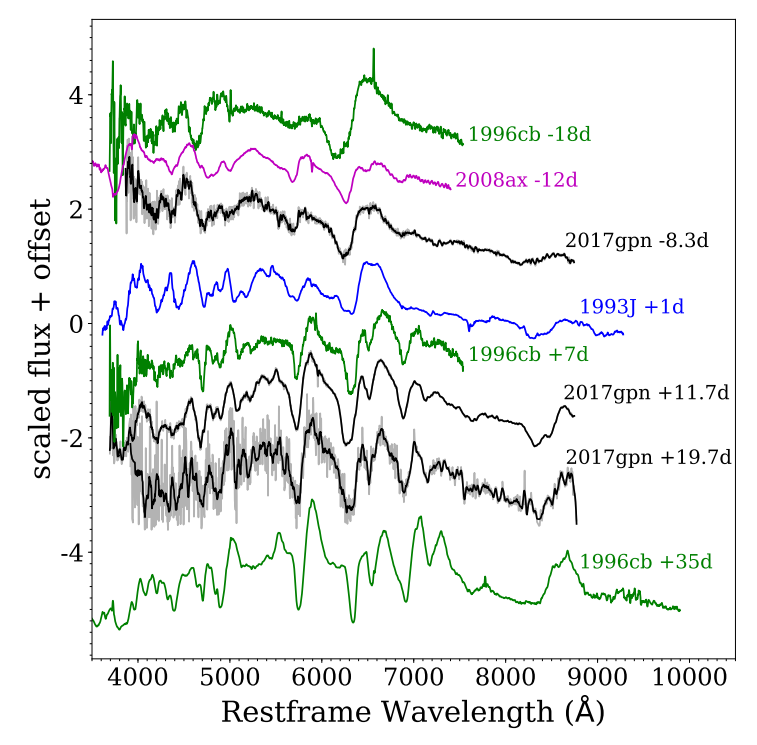

Figure 3. Three spectra of SN 2017gpn at different phases, the observation dates are indicated with respect to the $R$-band maximum light at $\mathrm{JD}=2458003.6$. Spectra of SNe IIb 1993J, 1996cb, and 2008ax are presented for comparison.

composition except for ${ }^{56} \mathrm{Ni}$. Since the amount and distribution of ${ }^{56} \mathrm{Ni}$ synthesized during the explosion plays a crucial role in the $\mathrm{SN}$ luminosity evolution, we consider two radial distributions for ${ }^{56} \mathrm{Ni}$. In the first one ${ }^{56} \mathrm{Ni}$ is totally mixed through the ejecta and in the second one ${ }^{56} \mathrm{Ni}$ falls off from the centre.

As input parameters for further hydrodynamical modelling, we varied the pre-SN star mass $\mathrm{M}$ and the radius $\mathrm{R}$, the mass of synthesized nickel $\mathrm{M}_{56} \mathrm{Ni}$, and the initial distribution of chemical elements in the pre-SN star.

\subsection{STELLA code}

To explode the hydrostatic non-evolutionary pre-SN models a onedimensional multifrequency radiation hydrocode STELLA is used. The full description of the code can be found in Blinnikov et al. (1998, 2006); a public version of STELLA is also included with the MESA distribution (Paxton et al. 2018). The STELLA code is used for the light-curve modelling of different types of $\mathrm{SNe}-$ Ia (Blinnikov et al. 2006), Ib/Ic (Folatelli et al. 2006; Tauris et al. 2013), IIb (Blinnikov et al. 1998; Tsvetkov et al. 2012), IIn (Chugai et al. 2004), IIP (Baklanov et al. 2005; Tominaga et al. 2009), Ic associated with long gamma-ray bursts (Volnova et al. 2017). The
Table 4. Parameters for the best-fitting and additional hydrodynamic models of SN 2017gpn.

\begin{tabular}{lcr}
\hline Parameter & Best-fitting model & Additional model \\
\hline $\mathrm{R}$ & $50 R_{\odot}$ & $400 R_{\odot}$ \\
$\mathrm{M}$ & $3.5 M_{\odot}$ & $3.5 M_{\odot}$ \\
$\mathrm{M}_{\mathrm{H} \_ \text {env }}$ & $0.06 M_{\odot}$ & $0.21 M_{\odot}$ \\
$\mathrm{M}_{\mathrm{CR}}$ & $1.41 M_{\odot}$ & $1.41 M_{\odot}$ \\
$\mathrm{M}_{5_{6} \mathrm{Ni}}$ & $0.11 M_{\odot}$, mixed & $0.11 M_{\odot}$, no mixing \\
$\mathrm{E}_{\text {exp }}$ & $1.2 \times 10^{51} \mathrm{erg}$ & $1.2 \times 10^{51} \mathrm{erg}$ \\
$\mathrm{t}_{\text {peak }, R}$ & $2017 \mathrm{Sept} 7.5$ & $2017 \mathrm{Sept} 5.6$ \\
\hline
\end{tabular}

STELLA code was compared with other well-known hydrodynamic codes and found to be in good agreement with them on the level of several per cent (e.g. Woosley et al. 2007; Kromer \& Sim 2009; Sim et al. 2010; Kozyreva et al. 2017; Tsang et al. 2020).

In the current calculations, we adopted 100 zones for the Lagrangian coordinate and 130 frequency bins. The explosion is initiated by putting thermal energy into the innermost layers. The energy is released in $0.1 \mathrm{~s}$, which is less than the hydrodynamic time of the pre-supernova. While this condition is true, the resulting light curve is not affected by the details of the explosion mechanism (Imshennik \& Nadezhin 1983).

\subsection{Best-fitting model}

To determine the best-fitting model of SN 2017gpn we consider a grid of parameters. The pre-SN mass varies between $3.5 M_{\odot}$ and $5.5 M_{\odot}$ with steps of $0.5 M_{\odot}$; the pre-SN radius and $\mathrm{E}_{\exp }$ take the values $\{50,100,200,400,600\} R_{\odot}$ and $\{0.6,1.2,2.4\} \times$ $10^{51} \mathrm{erg}$, respectively; three different $\mathrm{M}_{56} \mathrm{Ni}\{0.07,0.09,0.11\} M_{\odot}$ are considered, both with and without mixing. The mass of the hydrogen envelope $\mathrm{M}_{\mathrm{H} \_ \text {env }}$ is taken as $0.06 M_{\odot}$ which is in line with our expectations for Type IIb Supernovae.

After determination of the parameter grid we built trial models and chose the best-fitting model within the generated grids of light curves by calculating $\chi^{2}$ in the $R$ passband. The best-fitting model corresponds to the minimum value of $\chi^{2}$. We do not provide any statistical uncertainties, since this procedure requires enormous computational effort. Instead, the optimal model is recovered as a compromise between the fits to the observed light curve and the evolution of the velocity at the photosphere (see Section 4.2.1). The values of the best-fitting model parameters are summarized in Table 4. Fig. 2 compares the light curves of the model (solid lines) with the observations of SN 2017gpn.

In Fig. 4 we also show the distribution of the chemical elements and the density profile for a pre-SN star. Note that the best-fitting model shows a small amount of hydrogen in the pre-SN star composition, which is expected for SNe IIb (Filippenko et al. 1993). ${ }^{56} \mathrm{Ni}$ is totally mixed through the ejecta. 

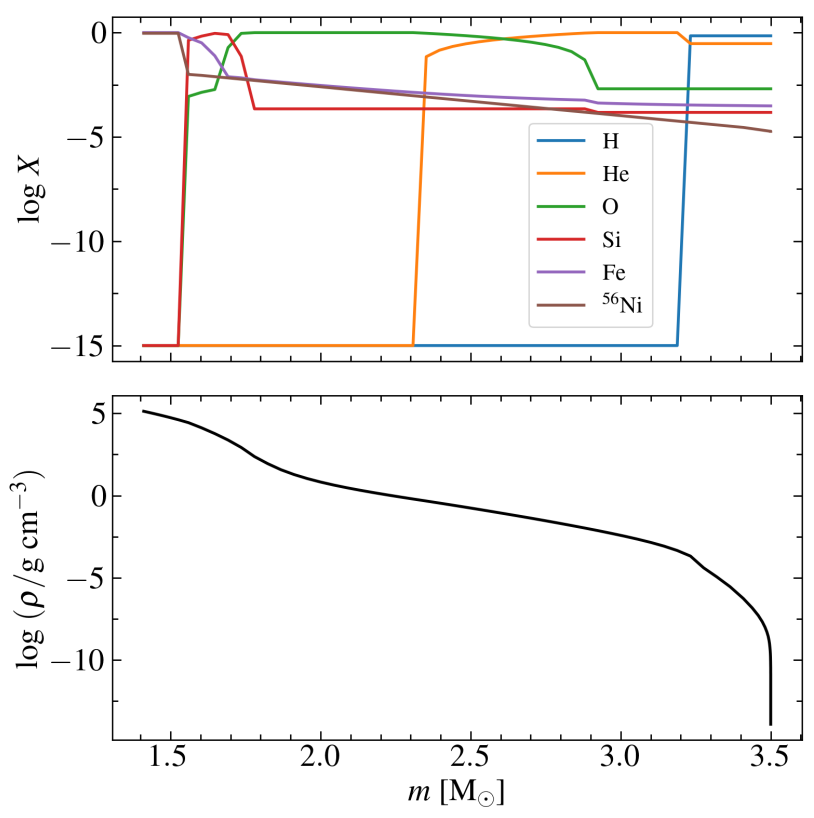

Figure 4. Mass fractions of the most abundant chemical elements in the ejecta (top) and density profile (bottom) for the best-fitting pre-SN star model with respect to the interior mass. The central region of $1.41 M_{\odot}$ is taken away.

\subsection{The influence of the model parameters on the light curve}

To provide a reasonable range of the best-fitting model parameters, we consider the dependence of the numerical LCs on an input parameter of the model while the others remain fixed. We vary the mass $\mathrm{M}$ and the radius $\mathrm{R}$ of the pre-SN star, the mass of synthesized ${ }^{56} \mathrm{Ni}$, and the energy of the explosion $\mathrm{E}_{\text {exp. }}$ In Fig. 5 we plot some modelled LCs in the $R$ passband that show a valid range for each parameter. All presented models are slightly shifted along the time axis to better describe the observational light curve.

The first considered parameter is the pre-SN mass $\mathrm{M}$, see Fig. 5 (a). This parameter mainly affects the width of the light curve, which becomes broader as the mass increases. This is explained by the fact that with a small mass the envelope becomes transparent faster. Thus, the LC increases before the maximum light and decreases rapidly after it. As Fig. 5 (a) shows, the range of valid pre-SN mass is $3-4 M_{\odot}$.

The next parameter is the amount of synthesized ${ }^{56} \mathrm{Ni}$ (Fig. 5 (b)). The models are brighter for higher ${ }^{56} \mathrm{Ni}$ masses. The LCs corresponding to the ${ }^{56} \mathrm{Ni}$ masses of 0.09 and $0.13 M_{\odot}$ lie below and above the best-fitting model light curve, respectively. These two values define the acceptance range of the $\mathrm{M}_{56} \mathrm{Ni}$ model parameter.

The pre-SN radius affects mainly the light-curve tail: a larger radius value corresponds to a brighter light curve after maximum light. The chosen range of the pre-SN radius is $20-70 R_{\odot}$; see Fig. 5 (c).

The last parameter that we vary is the explosion energy $E_{\text {exp }}$; see Fig. 5 (d). The determined range for the energy parameter is $(1.05-1.60) \times 10^{51} \mathrm{erg}$. As seen from Fig $5(\mathrm{~d})$, smaller values of $\mathrm{E}_{\text {exp }}$ correspond to brighter light curves. This dependence is in line with our expectations. A larger $\mathrm{E}_{\text {exp }}$, for a fixed mass of ${ }^{56} \mathrm{Ni}$ and fixed total mass, implies higher velocities and hence less trapping of gamma-ray photons. This leads to an increase in the predicted observed gamma-ray flux and, therefore, to a decrease in the emission in the visible light range.

\section{DISCUSSION}

\subsection{Comparison with other SNe IIb}

We collected data for well-studied SNe IIb with good photometric coverage in the $B$ and $R$ passbands, for which results of hydrodynamic modelling can be found in the literature. In Fig. 6 the light curves of selected $\mathrm{SNe}$ IIb are presented. It can be seen that LCs in the $B$ and $R$ passbands are similar - characteristic bell-shaped LCs. Moreover, as Pessi et al. (2019) showed, SNe Ilb take longer to reach maximum light and decline more quickly post-maximum than hydrogen-rich SNe II, so the authors assume that there is no continuum between $\mathrm{SNe}$ IIb and other SNe II like between SNe IIP and IIL types. SN 2017gpn has a typical SN IIb light curve, and belongs to one of the brightest well-studied SNe IIb: it is brighter than a typical member of Type IIb SN 1993J by 0.75 mag in the $R$ passband.

\subsubsection{Classification of Prentice \& Mazzali (2017)}

Following Prentice \& Mazzali (2017), stripped-envelope SNe should be subclassified into four groups: $\mathrm{Ib}, \mathrm{Ib}(\mathrm{II}), \mathrm{IIb}$, and IIb(I), using the additional parameters - equivalent width of $\mathrm{H} \alpha$ $\left(E W_{\mathrm{H} \alpha}\right)$ and $\mathrm{H} \alpha$ emission-to-absorption ratio $f_{e m} / f_{a b s}$. The $E W_{\mathrm{H} \alpha}$ parameter value is $>60 \AA$ for supernovae of group IIb(I), $20<E W_{\mathrm{H} \alpha}<60 \AA$ for $\mathrm{Ib}(\mathrm{II})$, and takes any reasonable value for groups Ib and IIb. The $\mathrm{H} \alpha$ emission-to-absorption ratio $f_{\text {em }} / f_{a b s}$ differs for groups IIb and IIb(I): it ranges from $0.3-1$ for $\mathrm{IIb}(\mathrm{I})$ and is greater than 1 for group IIb (see Fig. 7).

We calculated the intensity and equivalent width of $\mathrm{H} \alpha$ in our first spectrum ( $-8.3 \mathrm{~d}$ before $R$-band maximum) for SN 2017gpn and found $f_{e m} / f_{a b s}=0.63 \pm 0.04, E W_{\mathrm{H} \alpha}=123 \pm 3 \AA$. Therefore, SN 2017 gpn belongs to group $\mathrm{IIb}(\mathrm{I})$ which means that it might have less hydrogen in the envelope than most H-rich $\mathrm{SNe}$ such as 1993J, 2011fu, or 2011dh (see Table 5). However, it is similar to other $\mathrm{SNe} \mathrm{IIb}(\mathrm{I})-2008 \mathrm{ax}$ and 1996cb (the first position in a cross-correlation list according to SNID).

\subsubsection{Hydrodynamic models of other SNe IIb}

We compare the results of numerical simulations for SN 2017gpn and other SNe IIb (including groups IIb and $\mathrm{IIb}(\mathrm{I})$ of Prentice \& Mazzali 2017) presented in Fig. 6. Only hydrodynamic modelling of supernovae is chosen for comparison; we do not consider any analytical light-curve modelling or scaling to templates. The modelling results are summarised in Table 5 , where $\mathrm{M}_{\mathrm{CR}}$ is the mass of a compact object (generally this is a neutron star) and $\mathrm{M}_{\mathrm{ej}}$ is the mass of ejected matter.

The main modelling parameters such as the ejecta mass $\mathbf{M}_{\mathrm{ej}}$, the mass of ${ }^{56} \mathrm{Ni}, \mathrm{M}_{\mathrm{H} \_ \text {env }}$, and the explosion energy $\mathrm{E}_{\exp }$ are consistent with each other. An exception is the parameter of the pre-supernova radius $\mathrm{R}$. The considered hydrodynamic modelling shows that the pre-SN radius lies in a broad range from $30-$ $720 R_{\odot}$ and may be different for the same object in different models. For example, there are two models for SN 2008ax, one with a radius of $30-50 R_{\odot}$ (Folatelli et al. 2015) and another one with $\mathrm{R}=600 R_{\odot}$ (Tsvetkov et al. 2009). It should be noted 


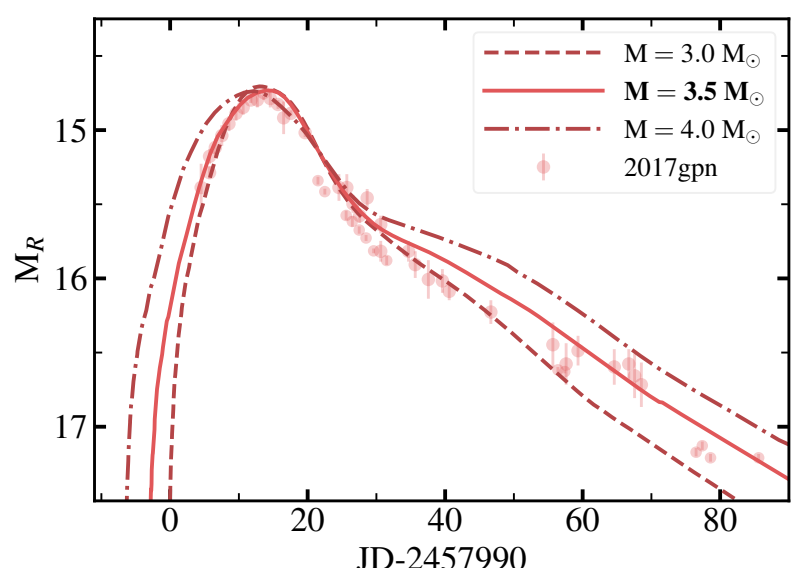

(a)

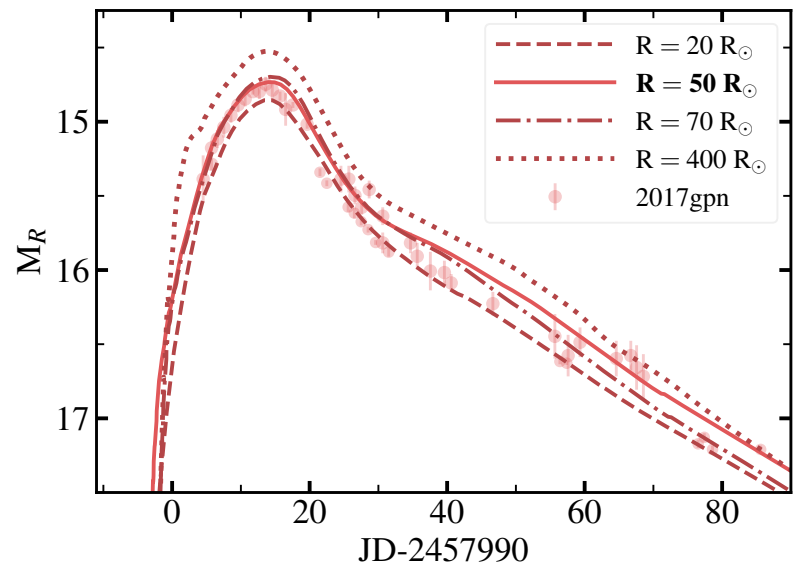

(c)

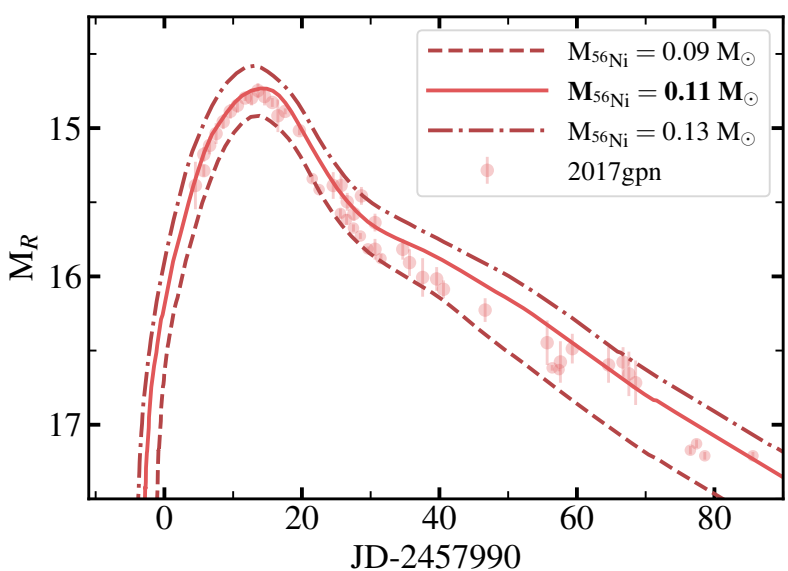

(b)

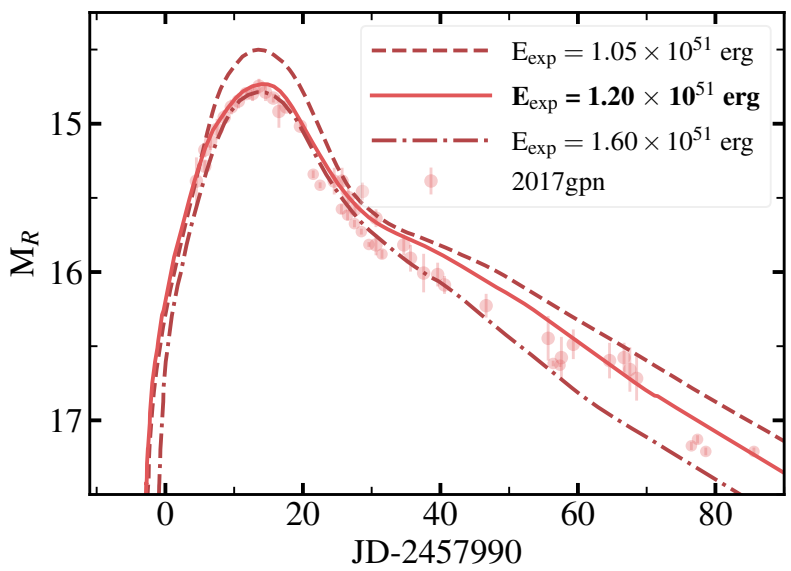

(d)

Figure 5. The dependence of the modelled $R$-passband LC on the pre-SN mass M (a), the amount of synthesized ${ }^{56} \mathrm{Ni}$ (b), the pre-SN radius R (c), and the explosion energy $E_{\exp }(\mathrm{d})$. All models are shifted along the time axis to better describe the observations. The best-fitting model is shown by a solid line in all plots; observations are shown by circles.
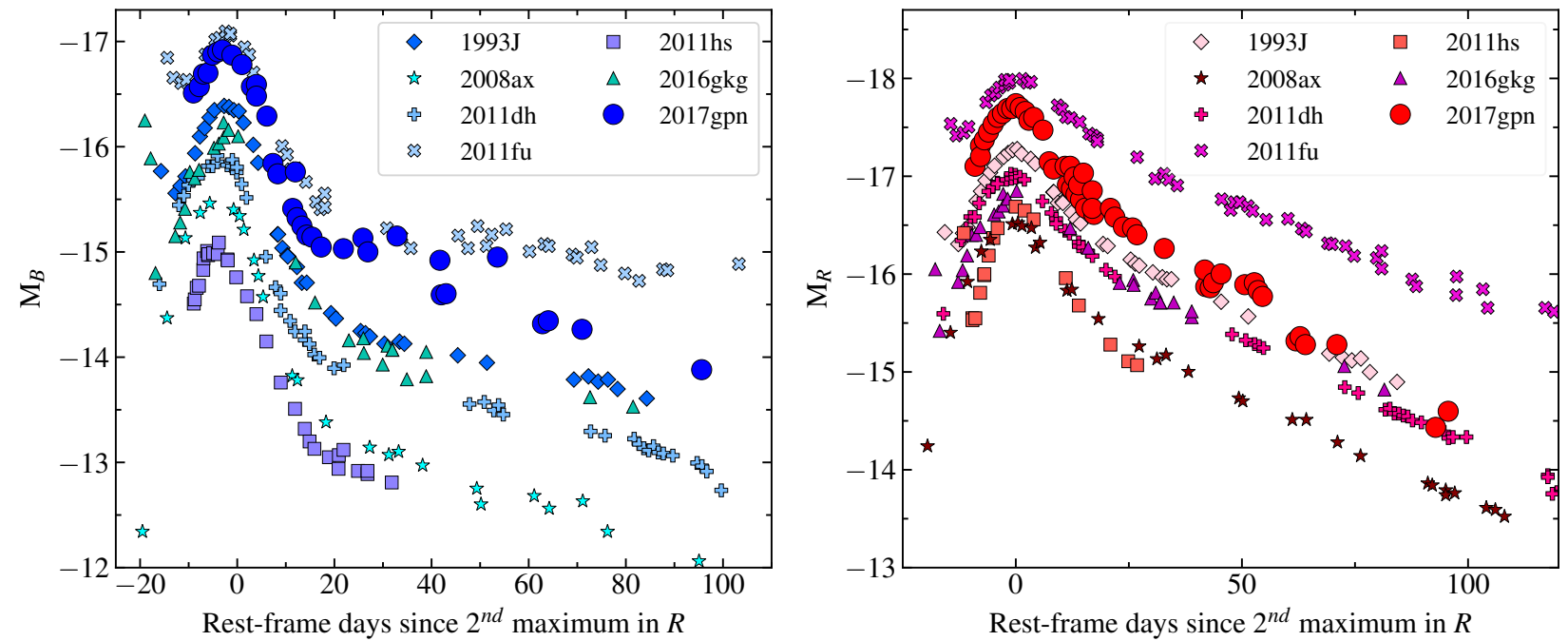

Figure 6. $\mathrm{M}_{B}$ and $\mathrm{M}_{R}$ light curves of SN 2017gpn in comparison with those of other Type IIb Supernovae: 1993J (Richmond et al. 1996), 2008ax (Tsvetkov et al. 2009), 2011dh (Tsvetkov et al. 2012), 2011fu (Kumar et al. 2013), 2011hs (Bufano et al. 2014), 2016gkg (Bersten et al. 2018). 


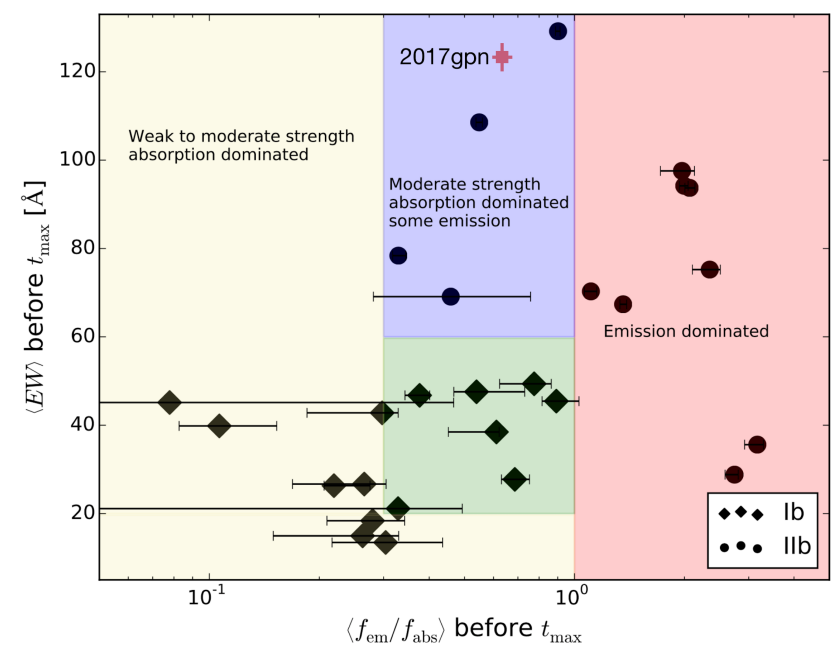

Figure 7. This figure is borrowed from Prentice \& Mazzali 2017 (fig. 7) with SN 2017gpn plotted (pink square). It illustrates the stripped-envelope supernovae subclassification based on the comparison of the line strength (equivalent width of $\mathrm{H} \alpha$ ) against the line profile ( $\mathrm{H} \alpha$ emission-to-absorption ratio $f_{e m} / f_{a b s}$ ) as proposed by Prentice \& Mazzali (2017). SN 2017gpn lies in the blue region that corresponds to group IIb(I). Groups IIb, Ib, and $\mathrm{Ib}(\mathrm{II})$ are in the red, yellow, and green regions, respectively.

that SN 2008ax belongs to the same group of IIb(I) supernovae as SN 2017gpn.

\subsection{Additional model}

Motivated by the discrepancy in the modelled radius for different $\mathrm{SNe}$ IIb, we have found another physically reasonable model for SN 2017 gpn with $\mathrm{R}=400 R_{\odot}$. For this additional model, radioactive nickel is located in the central part of the ejecta. We have also increased the mass of the hydrogen envelope to $0.21 M_{\odot}$, which is consistent with the fact that more extended SNe IIb should be also more H-rich (Prentice \& Mazzali 2017). The parameters of the additional model are listed in Table 4 . This model also well describes the observational data and agrees with the results of the hydrodynamic simulations for other SNe IIb.

There is no direct method to solve the inverse problem, i.e. to determine the parameters of the pre-supernova from the observational data. We can only build a model with given parameters and see how accurately it fits the data. Sometimes it can happen that models with different parameters reproduce observations equally well, as we see for our best-fitting and additional models (Fig. 2). However, if some additional information is available, e.g. observational photospheric velocities, we can compare our theoretical estimations with the observational values and make a choice between the models.

\subsubsection{Photospheric velocities}

Based on three spectra of SN 2017gpn obtained at different epochs with the Xinglong 2.16-m telescope, we measured the ejecta velocity from the $\mathrm{H} \alpha$ and $\mathrm{He}_{\mathrm{I}} \lambda 5876$ absorption lines (Table 3). In Fig. 8 we show the comparison between the velocities measured from these lines and theoretical values from the STELLA code, which are the velocities of the photosphere at the $\tau=2 / 3$ level in the $B$ band. The best-fitting model is consistent with the velocity

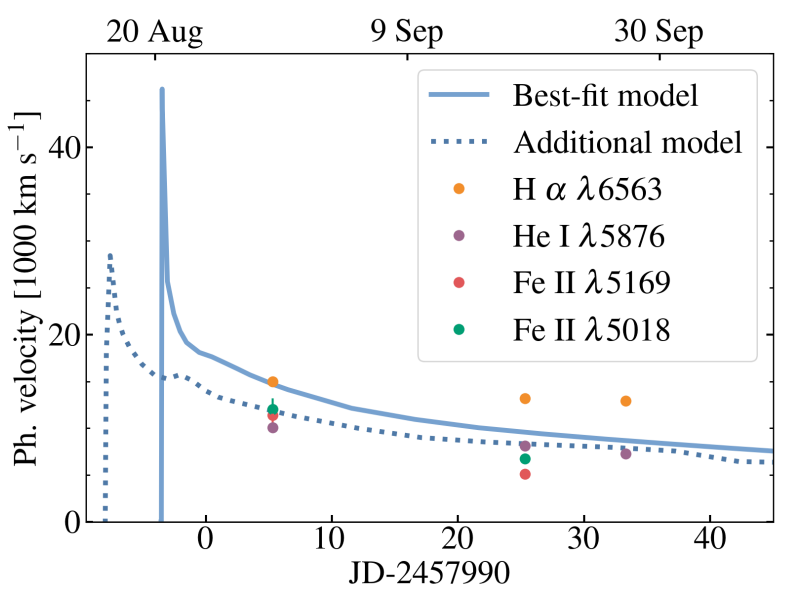

Figure 8. Photospheric velocity at the $\tau=2 / 3$ level as a function of time for the best-fitting model (solid line) and for the additional model of higher radius (dashed line); dots are the observational velocities measured from the H $\alpha, \mathrm{He}_{\text {I }} \lambda 25876, \mathrm{Fe}_{\text {II }} \lambda 5018$, and Fe II $\lambda 5169$ absorption lines.

measured from the $\mathrm{H} \alpha$ line for this epoch; the additional model is in good agreement with the $\mathrm{He}_{\mathrm{I}} \lambda 5876$ velocities for all three epochs.

It should be noted that $\mathrm{P}$ Cygni profiles are formed in all layers above the photosphere. Hence, the hydrogen and helium features do not necessarily reflect the photospheric velocities calculated by our hydrodynamic modelling. It has to be taken into account that the growth of the Sobolev optical depth (Sobolev 1960) at the photosphere level causes a significant blueshift of the P Cygni profile minimum, so the resulting velocity in that case will be overestimated (Kasen et al. 2002). This effect may explain why the velocities measured from the $\mathrm{H} \alpha$ line are greater than our theoretical estimates in Fig. 8. Meanwhile, according to Dessart \& Hillier 2005, 2006 the velocities measured from strong lines can be both smaller and larger than the photospheric ones.

Therefore, it is more reasonable to use "weak" lines, i.e. lines with small Sobolev optical depths, to estimate $v_{p h}$. (Dessart \& Hillier 2005) show that $\mathrm{Na}$ I D, Fe II $\lambda 5018, \mathrm{Fe}_{\text {II }} \lambda 5169$ are the most suitable lines to measure the photospheric velocities. We measured the velocities from the $\mathrm{Fe}$ II $\lambda \lambda 5018$ and 5169 lines for the first and second epochs of observations, the last epoch spectrum has a low signal-to-noise ratio to perform the measurements. We could not determine the velocities using $\mathrm{Na}$ I $\mathrm{D}$ features since they are close to $\mathrm{He}_{\mathrm{I}} \lambda 5876$ line, which is quite strong in $\mathrm{SNe} \mathrm{IIb}$.

The photospheric velocities derived for the additional model correspond slightly better to the velocities from the Fe II $\lambda \lambda 5018$ and 5169 lines for the first epoch of observations. For the second epoch the measured velocities are lower than the STELLA values for both models. Taking into account the modelling uncertainties, it is difficult to choose between the models based on these measurements only.

\subsection{2 $\quad{ }^{56} \mathrm{Ni}$ mixing}

From the theoretical bolometric LCs (Fig. 9) as well as LCs in filters (Fig. 2) it can be noticed that the light curve corresponding to the model with the uniform distribution of nickel behaves differently from the light curve that conforms with the model where nickel is concentrated in the centre of the ejecta. This is due to the fact that in the former case the radioactive decay energy contributes to the overall energy immediately after the explosion, whereas in the latter 


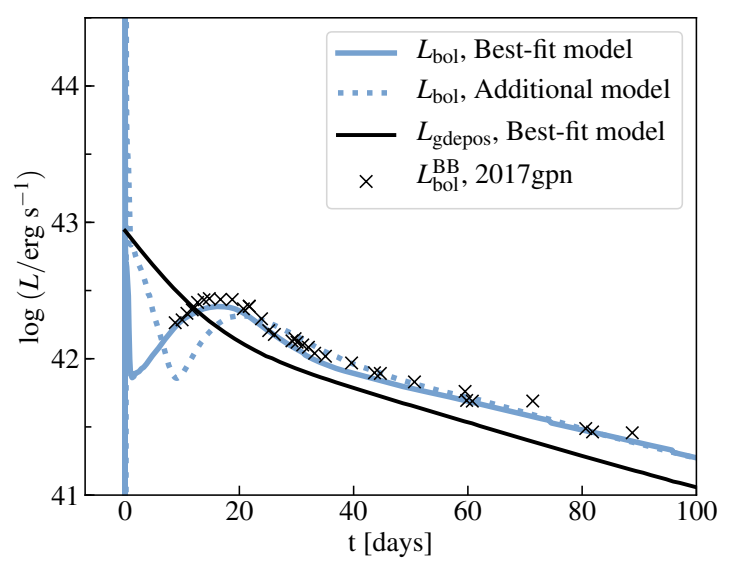

Figure 9. Theoretical bolometric light curves for the best-fitting (blue solid line) and the additional (blue dashed line) models of SN2017gpn. The crosses show the bolometric luminosity of SN 2017gpn calculated from $B$ and $R$ light curves with use of the SuperBol code (Nicholl 2018). The shift between the data and the best-fitting model is the same as in Fig. 2 but transformed to the rest frame. The black solid line is the power due to the gamma-ray deposition from ${ }^{56} \mathrm{Ni}$ and ${ }^{56} \mathrm{Co}$ decays for our best-fitting model. Accounting for the light travel time correction, $L_{\text {gdepos }}$ satisfies Arnett's law, going through the maximum of $L_{\mathrm{bol}}$.

case we observe two peaks in the light curve. The primary peak is associated with the heating of the outer layers of the star by the shock wave that is created by the rebound of the freely falling inner layers from the collapsed core. After that the envelope expands, cools, and therefore becomes transparent. The second peak is associated with the luminescence of the inner layers heated by the radioactive decays of ${ }^{56} \mathrm{Ni}$ and its products. For the additional model we fit the observed LCs by the second peak. Because of this, the best-fitting and additional models are shifted relative to each other in Fig. 2. The influence of ${ }^{56} \mathrm{Ni}$ mixing on the LC behaviour is also seen if we compare the additional model with the model in Fig 5 (c) (dotted line) with $\mathrm{R}=400 \mathrm{R}_{\odot}$ and ${ }^{56} \mathrm{Ni}$ totally mixed through the ejecta. Unlike the additional model, this model no longer describes the observations.

In Fig. 9 we also show the bolometric light curve of SN 2017gpn restored from the available photometry. To construct the bolometric light curve the SuperBol code is used (Nicholl 2018). To account for flux that is not covered by the observations, the black body extrapolation is applied. Even though we use only two passbands ( $B$ and $R$ ) the obtained bolometric LC agrees very well with our theoretical estimations.

\subsection{Arnett's law}

Arnett's law (Arnett 1982) states that the energy released on the surface at maximum light is equal to the energy deposed by gammaray radiation. This law is commonly used to estimate the amount of nickel produced in the explosion when the total luminosity at peak is known (Branch 1992). We plot the theoretical bolometric light curve and the curve corresponding to gamma-ray deposition from ${ }^{56} \mathrm{Ni}$ and ${ }^{56} \mathrm{Co}$ decays for our best-fitting model to check this law. As we can see from Fig. 9, the law is quite well satisfied; however, the power from gamma-ray deposition does not go directly through the $L_{\text {bol }}$ peak. This is explained by the fact that Arnett's law is not exact and in particular assumes an infinite speed of light. In the STELLA code the energy released in the centre will be "seen" with a delay of $R / c$, where $R$ is the radius of the expanding ejecta that changes with time and $c$ is the speed of light. The observed difference increases towards the tail since the radius increases as well.

\subsection{SN 2017gpn position relative to the host galaxy centre}

Supernova 2017gpn exploded in the spiral galaxy NGC 1343 at a projected distance of $D \simeq 21 \mathrm{kpc}$ from its centre (see Fig. 1). Such location is unusual for core-collapse supernovae, in particular for Type IIb, since it is believed that stripped-envelope CCSNe are formed from very massive stars in star-formation regions of galaxies (see Audcent-Ross et al. 2020 and references therein). Assuming that SN 2017gpn belongs to the galactic disk we can take into account the projection effect. The deprojected distance $D_{\text {dep }}$ between the supernova and the host centre is calculated as

$D_{\text {dep }}=D \sqrt{\cos ^{2} \alpha+\sin ^{2} \alpha \sec ^{2} i}$,

where $\alpha$ is the angle between the projected distance and the major axis of a galaxy and $i$ is the disc inclination angle. According to HyperLEDA $i$ equals $67.3 \mathrm{deg}$ and the major axis position angle of NGC 1343 is $78.8 \mathrm{deg}$ (Makarov et al. 2014). Using these values and the coordinates of SN 2017gpn and its host galaxy centre we can calculate the deprojected distance for SN 2017gpn, which is $\sim 52 \mathrm{kpc}$. To understand how exceptional this position is we study the absolute and relative separations between the supernova positions and their host galaxy centres for a sample of SNe IIb.

Hereafter, by the distance between a supernova and its host galaxy we mean the projection of the distance onto the picture plane, which is obviously smaller than the real distance. However, the star-evolution theory predicts that CCSNe including $\mathrm{SNe} \mathrm{IIb}$ mainly appear in the galactic planes of spiral galaxies, in regions of high star-formation rate. Therefore, we assume that the contribution of the projection onto the line of sight is relatively small and this underestimation of the distance does not significantly affect our analysis.

We collected 71 confirmed SNe IIb and 108 candidates for SNe IIb from the Open Supernova Catalog (Guillochon et al. 2017). The confirmed SNe IIb are supernovae for which multiple spectra have been obtained and a detailed spectral analysis has been performed. If only a spectrum is available (usually single spectroscopic confirmation following the astronomical telegram about the transient discovery) we consider a supernova as a SN IIb candidate.

First, we calculated the absolute galactocentric distance $D$ for each object as $D \simeq d_{a} \times \Theta$. The angle $\Theta$ is the angle between the supernova and the host galaxy centre. The angular distance $d_{a}$ for flat $\Lambda \mathrm{CDM}$ cosmology with $\Omega_{\Lambda}=0.7$ and $H_{0}=70 \mathrm{~km} \mathrm{~s}^{-1} \mathrm{Mpc}^{-1}$ is

$d_{a}=\frac{c}{H_{0} \times(1+z)} \int_{0}^{z} \frac{d z^{\prime}}{\sqrt{\left(1-\Omega_{\Lambda}\right) \times\left(1+z^{\prime}\right)^{3}+\Omega_{\Lambda}}}$,

where $z$ is the redshift and $c$ is the speed of light. The distribution of Type IIb Supernovae by $D$ is presented in Fig. 10. Most SNe IIb, about 85 per cent, are located inside a radius of $12 \mathrm{kpc}$. However, there is a local maximum near $20 \mathrm{kpc}$, which may be due to the fact that the radius of galaxies can vary widely.

To perform a more accurate analysis we determined the SNhost separation relative to the host size. To characterise the size of a galaxy we used a $D_{25}$ value, which is the major diameter measured tothe $B$-passband $25 \mathrm{mag} \operatorname{arcsec}^{-2}$ isophote. The $D_{25}$ values were 
Table 5. Comparison of the hydrodynamic modelling results for different SNe IIb.

\begin{tabular}{lccccccr}
\hline SN name & $\begin{array}{c}\mathrm{M}_{\mathrm{CR}} \\
{\left[\mathrm{M}_{\odot}\right]}\end{array}$ & $\begin{array}{c}\mathrm{M}_{\mathrm{ej}} \\
{\left[\mathrm{M}_{\odot}\right]}\end{array}$ & $\begin{array}{c}\mathrm{M}_{56} \mathrm{Ni} \\
{\left[\mathrm{M}_{\odot}\right]}\end{array}$ & $\begin{array}{c}\mathrm{M}_{\mathrm{H} \_ \text {env }} \\
{\left[\mathrm{M}_{\odot}\right]}\end{array}$ & $\begin{array}{c}\mathrm{R} \\
{\left[\mathrm{R}_{\odot}\right]}\end{array}$ & $\begin{array}{c}\mathrm{E}_{\exp } \\
{\left[10^{51} \mathrm{erg}\right]}\end{array}$ & Reference \\
\hline 1993J & $\sim 1.4$ & $1.4-3.1$ & $0.06-0.08$ & 0.2 & $430-720$ & 1.2 & $\begin{array}{r}\text { Woosley et al. (1994) } \\
\text { Blinnikov et al. (1998) }\end{array}$ \\
\hline $2008 \mathrm{ax}$ & 1.41 & 2.39 & 0.11 & - & 600 & 1.5 & $\begin{array}{r}\text { Tsvetkov et al. (2009) } \\
\text { Folatelli et al. (2015) }\end{array}$ \\
\hline $2008 \mathrm{ax}$ & 1.5 & $1.8-3.5$ & $0.05-0.07$ & 0.06 & $30-50$ & $0.8-1.2$ & Bufano et al. (2014) \\
\hline $2011 \mathrm{hs}$ & 1.5 & $1.5-2.5$ & 0.04 & $<0.5$ & $500-600$ & 0.85 & Morales-Garoffolo et al. (2015) \\
\hline $2011 \mathrm{fu}$ & 1.5 & 3.5 & 0.15 & 0.3 & 450 & 1.3 & Tsvetkov et al. (2012) \\
$2011 \mathrm{dh}$ & 1.41 & $2.24-4.24$ & 0.07 & - & $150-300$ & $2-4$ & Bersten et al. (2012) \\
\hline $2016 \mathrm{gkg}$ & 1.5 & 2 & 0.06 & 0.1 & 200 & $0.6-1$ & $\begin{array}{r}\text { Piro et al. (2017) } \\
2016 \mathrm{gkg}\end{array} 1.5-1.6$ \\
\hline
\end{tabular}

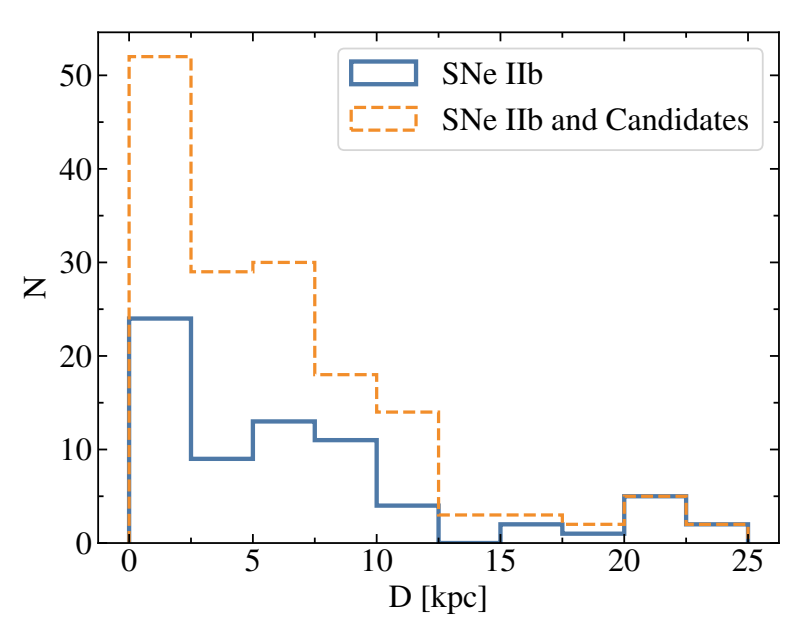

Figure 10. Histogram of the supernovae distribution depending on the projection of the distance between SN and its host galaxy centre, $D$. The orange dashed line corresponds to all considered supernovae including confirmed $\mathrm{SNe} \mathrm{IIb}$ and candidates for $\mathrm{SNe} \mathrm{IIb}$. The solid blue line corresponds to the distribution of confirmed SNe IIb only.

extracted from the HyperLEDA extragalactic data base (Makarov et al. 2014).

The full list of studied supernovae and the absolute and relative distances are summarised in Table A1: the first column is the number in the list for easier searching, the second column consists of the supernova names starting with confirmed $\mathrm{SNe} \mathrm{IIb}$, and continuing with SNe IIb candidates. The equatorial coordinates (RA, Dec.) of supernovae and their host galaxies are presented in the third, fourth, fifth and sixth columns, respectively. The seventh column indicates the redshift $z$. $D_{25}$ is given in column eight. The angle $\Theta$ expressed in arcsec is shown in the ninth column. Columns 10 and 11 contain the absolute distance $D$ in kpc and relative separation normalized to the size of the host galaxies, respectively.

In Fig. 11 we present the relative separation between $\mathrm{SNe}$ and their host galaxies depending on the redshift. To evaluate how far away a supernova is, we chose a value of 1 for the relative separation, which is shown by the horizontal green line in Fig. 11. There are eight $\mathrm{SNe}$ IIb that lie above the solid green line; we collect them

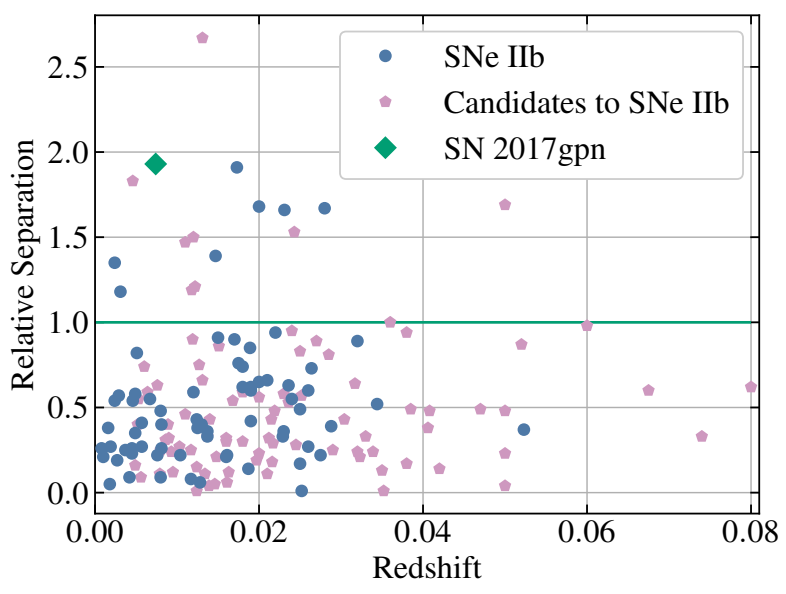

Figure 11. Relative separation between supernovae and their host galaxy centres as a function of redshift. Confirmed SNe IIb are plotted in blue dots; candidates for SNe IIb, in pink pentagons; and the studied SN 2017gpn is marked with the green diamond. SNe above the green line are considered to be distant from the centres of their hosts.

into a group of distant supernovae. SN $2017 \mathrm{gpn}$ is the most distant from the host galaxy centre among the confirmed SNe IIb.

After that, we collected images for all these distant $\mathrm{SNe}$ with the goal of investigating their unexpected location (see Fig. 12). The majority of them are in continuations of spiral arms, e.g. supernovae $1997 \mathrm{dd}$ or $2001 \mathrm{cf}$. Exceptions are supernovae $2011 \mathrm{ft}$ and $2017 \mathrm{gpn}$, which are well outside the borders of their host galaxies. We found Pan-STARRS1 images (Chambers et al. 2016; Flewelling et al. 2016) for SN 2011ft in the $r, i, z$ and $y$ passbands where one can notice a diffuse red object exactly at the SN $2011 \mathrm{ft}$ position, which can be associated with the host galaxy of SN $2011 \mathrm{ft}$.

In addition, we consider the object with the highest relative separation in Fig. 11 (rel. sep. is 2.67; see Table A1), SN 2017ati, a candidate for type IIb SNe. It turns out that this SN exploded in a system of interacting galaxies. Due to this interaction, a region with a high star-formation rate could be formed, and this explains the detection of the core-collapse supernova far from the host galaxy disc. Therefore, SN 2017gpn is the only distant SNe that is not located in a region with a high star-formation rate. 

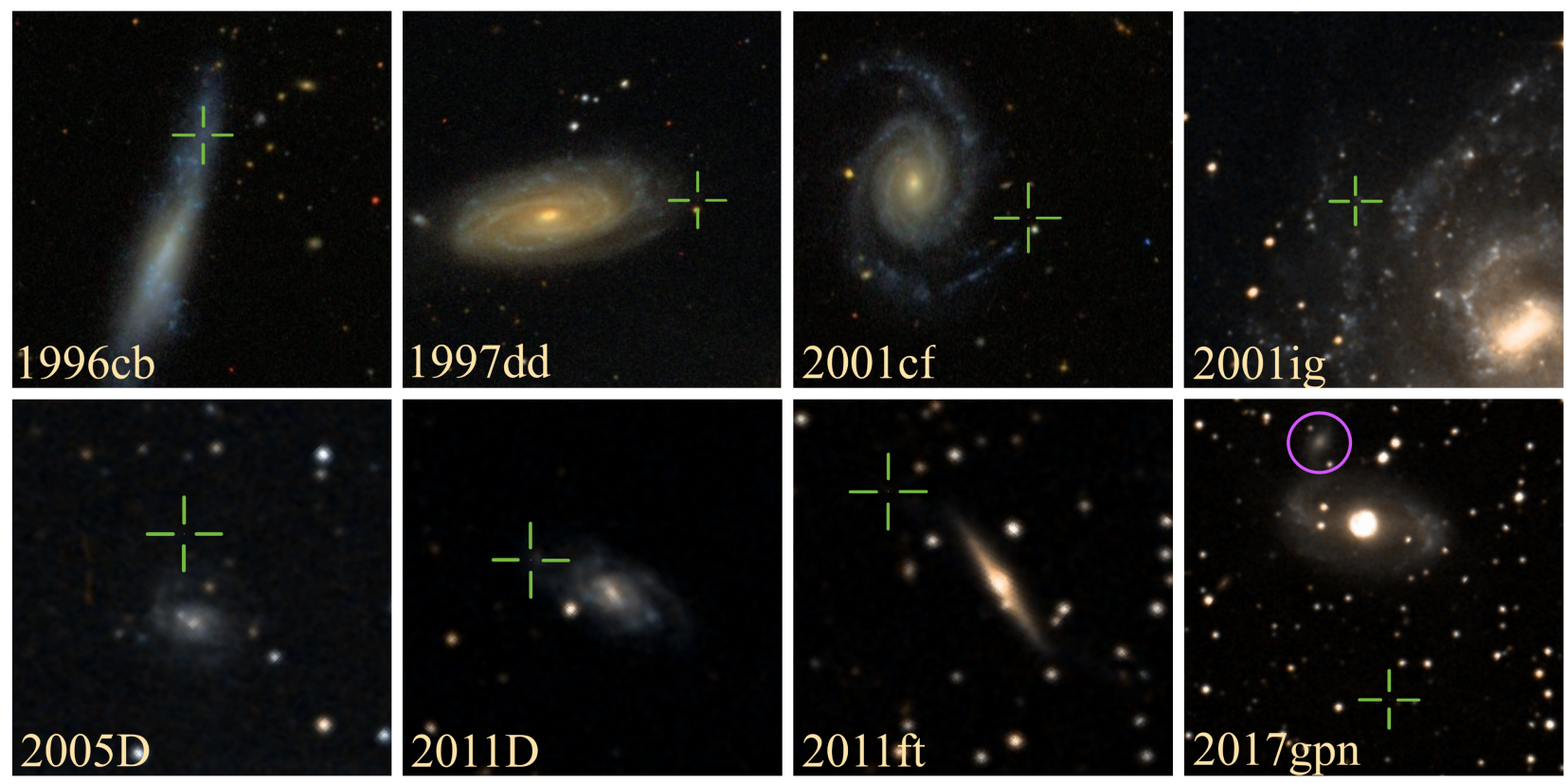

Figure 12. Optical images of supernovae distant from their host galaxy centres. SNe are marked by green crosses. All images were provided by SDSS (Blanton et al. 2017; Gunn et al. 1998) and DSS. The purple circle denotes the galaxy ZOAG G134.74+13.65.

According to the stellar evolution theory, the progenitor star of SN IIb should be a massive star with an initial mass of $\sim 30 M_{\odot}$. The fact that SN 2017gpn exploded far from a region with a high starformation rate challenges this popular scenario. We have considered three different hypotheses to explain its location.

First, the progenitor of SN 2017gpn could be a superspeed star. Brown et al. (2005) have discovered a hypervelocity star SDSS J090745.0+024507 with a mass of $\sim 4 M_{\odot}$ ejected from the Milky Way centre and left with a velocity of $709 \mathrm{~km} \mathrm{~s}^{-1}$. If we presume that the SN 2017gpn progenitor mass is about $30 M_{\odot}$, the average lifetime of such a star will be $\sim 3 \mathrm{Myr}$ calculated by the formula $t_{\text {lif } e} \simeq\left(\frac{M_{\odot}}{M_{\text {star }}}\right)^{2}$. If it moves at a speed of $1000 \mathrm{~km} \mathrm{~s}^{-1}$ (Hills 1988), it could move away from the centre of the host galaxy by $\sim 29 \mathrm{kpc}$ during its lifetime. However, such a high velocity implies that the kinetic energy is $\sim 3 \times 10^{50} \mathrm{erg}$; therefore an effective mechanism of star acceleration is required.

The second hypothesis is that part of the spiral arm of the host galaxy NGC 1343 is faint and therefore cannot be easily observed. For example, a similar situation is observed for the object AM 1316241 (Keel \& White 2001; see Fig. 13). In this case we can see the faint spiral arm of the galaxy only because it is illuminated by the light of a background elliptical galaxy. It is important that this part of the spiral structure does not lie on the continuation of the bright spiral arm; therefore, a SN explosion there (in the absence of a "lamp" behind) will appear to be outside the galaxy.

The third hypothesis is that the host galaxy of SN 2017gpn experienced an interaction with other galaxies in the past. Tidal force destroyed the satellite galaxy and provided enough gas, which could condense far from the NGC 1343 centre. Moreover, we can see the interaction between the galaxy ZOAG G134.74+13.65 and the SN 2017gpn host galaxy, which could also cause the formation of gas clouds with a high star-formation rate (see Fig. 12, panel 8).

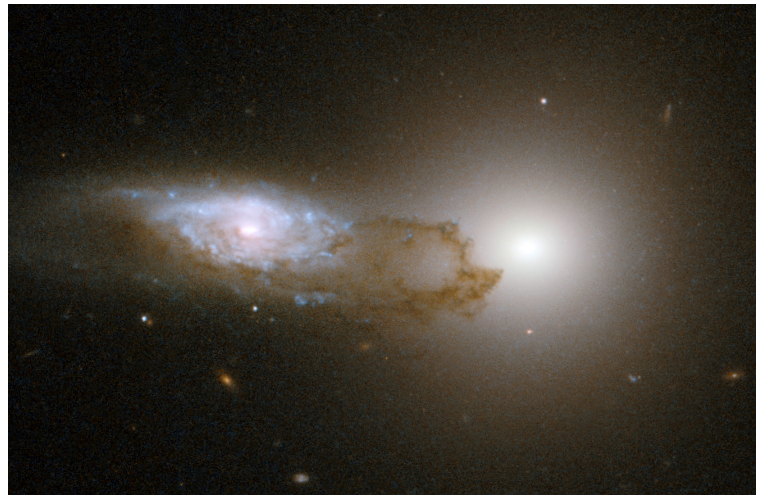

Figure 13. An image of AM 1316-241 obtained by the Hubble Space Telescope (Keel \& White 2001). The faint spiral arms are visible owing to the light from the background elliptical galaxy.

\subsection{Connection with G299232}

Initially SN 2017gpn was considered as a possible optical counterpart of the GW event G299232 since it was discovered $2 \mathrm{~d}$ later in its error-box ${ }^{4}$. If we assume that gravitational energy is released by a collapse, GW events are expected from supernova explosions (Herant et al. 1994) and could be detected by the LIGO/Virgo experiment (The LIGO Scientific Collaboration et al. 2019).

Nevertheless, the results of the hydrodynamic modelling show that the explosion happened on Aug 20 ( $\sim 3.5 \mathrm{~d}$ before the GW alert) following the best-fitting model, or on Aug 17 for the additional model, i.e. $\sim 8 \mathrm{~d}$ before registration of G299232. G299232 is a lowsignificance event, so it could be a false signal; even if it is not, it is still implausible that SN 2017gpn could be associated with this

\footnotetext{
4 https://gcn.gsfc.nasa.gov/other/G299232.gcn3
} 
alert. Neither of our calculated models favor the electromagnetic counterpart of the gravitational event.

\section{CONCLUSIONS}

In this paper we have presented spectroscopic and photometric observations of the Type IIb Supernova 2017gpn and the results of the numerical modelling of its $B, R$ light curves with the STELLA code. The best-fitting hydrodynamic model has the following parameter values: pre-SN radius $50 R_{\odot}$, pre-SN mass $3.5 M_{\odot}$, mass of synthesized nickel totally mixed in the envelope $0.11 M_{\odot}$, mass of the compact remnant $1.41 M_{\odot}$ (i.e. neutron star as a remnant), and energy of the explosion $1.2 \times 10^{51} \mathrm{erg}$. We also determined the ranges for these parameters by considering the dependence of the modelled light curves on each parameter while the others remain fixed. The obtained ranges are 3-4 $M_{\odot}$ for the pre-SN mass, 20 $70 R_{\odot}$ for the pre-SN radius, $0.09-0.13 M_{\odot}$ for the mass of ${ }^{56} \mathrm{Ni}$, and, finally, $(1.05-1.60) \times 10^{51} \mathrm{erg}$ for $\mathrm{E}_{\mathrm{exp}}$.

The study of Type IIb Supernovae is an important part of the exploration of the chemical composition of the Universe. The nucleosynthesis yields of CCSNe including SNe IIb are characterized by strong contributions to the so-called alpha elements $\mathrm{O}, \mathrm{Ne}, \mathrm{Mg}, \mathrm{Si}$, S, Ar, Ca, and Ti (Thielemann et al. 2018) and the heavy elements, namely $\mathrm{Ni}$, Co and Fe.

According to the Open Supernova Catalog (Guillochon et al. 2017) only about a couple of dozen SNe IIb have detailed photometry that allows the performance of reliable hydrodynamic modelling. Some of these SNe are considered in this paper and compared with SN 2017gpn taking into account a physically motivated classification of stripped-envelope SNe proposed by Prentice \& Mazzali (2017). In this classification SN 2017gpn belongs to the group IIb(I) which is characterized by strong hydrogen line profiles before maximum light, which weaken greatly over time, and $\mathrm{H} \alpha$ P Cygni profile dominated by the absorption component. Analysis of the hydrodynamic modelling results of different $\mathrm{SNe}$ IIb shows that the mass of synthesised ${ }^{56} \mathrm{Ni}$ is in the range $0.05-0.15 M_{\odot}$.

The modelling results for SN $2017 \mathrm{gpn}$ are consistent with those for SNe IIb considered, especially if we compare them with the modelling results for SN 2008ax which is of IIb(I) group according to Prentice \& Mazzali (2017). These results together with the observational data presented here contribute to the study of the Type IIb SN phenomenon, increasing the sample of well-studied SNe IIb.

Finally, we considered three different hypotheses that could explain SN 2017gpn's distant location relative to its host galaxy:

- the progenitor of SN 2017gpn is a hypervelocity star ejected by NGC 1343 with an average speed more than $1000 \mathrm{~km} \mathrm{~s}^{-1}$;

- the progenitor exploded in a faint spiral arm of the host galaxy;

- the progenitor is formed in a region of interaction between the host galaxy and another galaxy in the past.

However, there is still a chance that the SN 2017gpn progenitor was not a massive star exploding for some reason far from regions of high star-formation rate. A similar idea was proposed for the Type Ibn Supernova PS1-12sk by Hosseinzadeh et al. (2019). This question is open and challenges modern star-evolution models.

\section{ACKNOWLEDGEMENTS}

EAB acknowledges support from a Russian Science Foundation grant 18-72-00159 for studying the question of the distant posi- tion of SN 2017gpn relative host galaxy centre. SIB and MVP acknowledge support from a Russian Science Foundation grant 18-12-00522 for supernova modelling with the STELLA code. The authors acknowledge the support from the Program of Development of M.V. Lomonosov Moscow State University (Leading Scientific School "Physics of stars, relativistic objects and galaxies"). The authors are grateful to O. I. Spiridonova and the Zeiss1000 staff for help with observations. We thank K. L. Malanchev and M. Sh. Potashov for helpful discussions. XW is supported by the National Natural Science Foundation of China (NSFC grants 11325313, 11633002, and 11761141001), and the National Program on Key Research and Development Project (grant no. 2016YFA0400803). We acknowledge the support of the staff of the Xinglong 2.16-m telescope. This work was partially supported by the Open Project Program of the Key Laboratory of Optical Astronomy, National Astronomical Observatories, Chinese Academy of Sciences. This research has made use of NASA's Astrophysics Data System Bibliographic Services and the following Python software packages: NUMPY (van der Walt et al. 2011), MATPLOTLIB (Hunter 2007), ASTROPY (Astropy Collaboration et al. 2013; Price-Whelan et al. 2018).

\section{DATA AVAILABILITY}

The data underlying this article are available in the article.

\section{REFERENCES}

Anderson J. P., et al., 2014, ApJ, 786, 67

Arnett W. D., 1982, ApJ, 253, 785

Astropy Collaboration et al., 2013, A\&A, 558, A33

Audcent-Ross F. M., Meurer G. R., Audcent J. R., Ryder S. D., Wong O. I., Phan J., Williamson A., Kim J. H., 2020, MNRAS, 492, 848

Baklanov P. V., Blinnikov S. I., Pavlyuk N. N., 2005, Astronomy Letters, 31, 429

Barbon R., Benetti S., Cappellaro E., Patat F., Turatto M., Iijima T., 1995, A\&AS, 110, 513

Bersten M. C., et al., 2012, ApJ, 757, 31

Bersten M. C., et al., 2018, Nature, 554, 497

Blanton M. R., et al., 2017, AJ, 154, 28

Blinnikov S. I., Bartunov O. S., 1993, A\&A, 273, 106

Blinnikov S. I., Eastman R., Bartunov O. S., Popolitov V. A., Woosley S. E., 1998, ApJ, 496, 454

Blinnikov S. I., Röpke F. K., Sorokina E. I., Gieseler M., Reinecke M., Travaglio C., Hillebrandt W., Stritzinger M., 2006, A\&A, 453, 229

Blondin S., Tonry J. L., 2007, ApJ, 666, 1024

Branch D., 1992, ApJ, 392, 35

Brown W. R., Geller M. J., Kenyon S. J., Kurtz M. J., 2005, ApJ, 622, L33 Bufano F., et al., 2014, MNRAS, 439, 1807

Caimmi M., 2017, Transient Name Server Discovery Report, 2017-973, 1

Chambers K. C., et al., 2016, arXiv e-prints,

Chugai N. N., et al., 2004, MNRAS, 352, 1213

Clocchiatti A., Wheeler J. C., 1997, ApJ, 491, 375

Copperwheat C. M., et al., 2017, GRB Coordinates Network, 21755, 1

Dessart L., Hillier D. J., 2005, A\&A, 439, 671

Dessart L., Hillier D. J., 2006, A\&A, 447, 691

Filippenko A. V., 1997, ARA\&A, 35, 309

Filippenko A. V., Matheson T., Ho L. C., 1993, ApJ, 415, L103

Flewelling H. A., et al., 2016, arXiv e-prints,

Folatelli G., et al., 2006, ApJ, 641, 1039

Folatelli G., Bersten M. C., Kuncarayakti H., Benvenuto O. G., Maeda K., Nomoto K., 2015, ApJ, 811, 147

Guillochon J., Parrent J., Kelley L. Z., Margutti R., 2017, ApJ, 835, 64

Gunn J. E., et al., 1998, AJ, 116, 3040 
Hakobyan A. A., Adibekyan V. Z., Aramyan L. S., Petrosian A. R., Gomes J. M., Mamon G. A., Kunth D., Turatto M., 2012, A\&A, 544, A81

Hakobyan A. A., et al., 2016, MNRAS, 456, 2848

Herant M., Benz W., Hix W. R., Fryer C. L., Colgate S. A., 1994, ApJ, 435, 339

Hills J. G., 1988, Nature, 331, 687

Hoflich P., Langer N., Duschinger M., 1993, A\&A, 275, L29

Holmes S., et al., 2011, Publications of the Astronomical Society of the Pacific, 123, 1177

Hosseinzadeh G., McCully C., Zabludoff A. I., Arcavi I., French K. D., Howell D. A., Berger E., Hiramatsu D., 2019, ApJ, 871, L9

Hunter J. D., 2007, Computing in Science and Engineering, 9, 90

Imshennik V. S., Nadezhin D. K., 1983, Soviet Scientific Reviews, Section E: Astrophysics and Space Physics Reviews, 2, 75

Jonker P. G., Fraser M., Nissanke S., Fender R. P., Broderick J., Rowlinson A., Wijers R. A. M. J., Stappers B., 2017, GRB Coordinates Network, 21737, 1

Kasen D., Branch D., Baron E., Jeffery D., 2002, ApJ, 565, 380

Keel W. C., White R. E., 2001, ApJ, 121, 1442

Komarov V. V., Komarova V. N., Moskvitin A. S., Drabek S. V., Emelyanov E. V., Shergin V. S., 2020, Astronomical Journal of Azerbaijan, p. in press

Kozyreva A., et al., 2017, MNRAS, 464, 2854

Kromer M., Sim S. A., 2009, MNRAS, 398, 1809

Kumar B., et al., 2013, MNRAS, 431, 308

Landolt A. U., 1992, AJ, 104, 340

Lipunov V., et al., 2010, Advances in Astronomy, 2010, 349171

Lipunov V. M., et al., 2017, GRB Coordinates Network, 21719, 1

Makarov D., Prugniel P., Terekhova N., Courtois H., Vauglin I., 2014, A\&A, 570, A13

Modjaz M., et al., 2014, AJ, 147, 99

Morales-Garoffolo A., et al., 2015, MNRAS, 454, 95

Nadyozhin D. K., Razinkova T. L., 1986, Nauchnye Informatsii, 61, 29

Nicholl M., 2018, Research Notes of the AAS, 2, 230

Nomoto K., Suzuki T., Shigeyama T., Kumagai S., Yamaoka H., Saio H., 1993, Nature, 364, 507

Paxton B., et al., 2018, ApJS, 234, 34

Pessi P. J., et al., 2019, MNRAS, 488, 4239

Piro A. L., Muhleisen M., Arcavi I., Sand D. J., Tartaglia L., Valenti S., 2017, ApJ, 846, 94

Prentice S. J., Mazzali P. A., 2017, MNRAS, 469, 2672

Price-Whelan A. M., et al., 2018, AJ, 156, 123

Qiu Y., Li W., Qiao Q., Hu J., 1999, AJ, 117, 736

Richmond M. W., Treffers R. R., Filippenko A. V., Paik Y., 1996, AJ, 112, 732

Roberts D., Kolb U., 2018, 2

Rui L., et al., 2017, The Astronomer's Telegram, 10681

Sanders N. E., et al., 2015, The Astrophysical Journal, 799, 208

Schlafly E. F., Finkbeiner D. P., 2011, ApJ, 737, 103

Sim S. A., Kromer M., Röpke F. K., Sorokina E. I., Blinnikov S. I., Kasen D., Hillebrandt W., 2010, in Pogorelov N. V., Audit E., Zank G. P., eds, Astronomical Society of the Pacific Conference Series Vol. 429, Numerical Modeling of Space Plasma Flows, Astronum-2009. p. 148 (arXiv: 0911.1549)

Sobolev V. V., 1960, Moving envelopes of stars

Springob C. M., Haynes M. P., Giovanelli R., Kent B. R., 2005, ApJS, 160, 149

Stetson P. B., 1987, in Bulletin of the American Astronomical Society. p. 745

Tauris T. M., Langer N., Moriya T. J., Podsiadlowski P., Yoon S.-C., Blinnikov S. I., 2013, ApJ, 778, L23

The LIGO Scientific Collaboration et al., 2019, arXiv e-prints, p. arXiv: 1908.03584

Thielemann F.-K., Isern J., Perego A., von Ballmoos P., 2018, Space Sci. Rev., 214, 62

Tominaga N., Blinnikov S., Baklanov P., Morokuma T., Nomoto K., Suzuki T., 2009, ApJ, 705, L10

Tsang B. T. H., Goldberg J. A., Bildsten L., Kasen D., 2020, arXiv e-prints, p. arXiv:2006.01832
Tsvetkov D. Y., Volkov I. M., Baklanov P., Blinnikov S., Tuchin O., 2009, Peremennye Zvezdy, 29

Tsvetkov D. Y., Volkov I. M., Sorokina E., Blinnikov S., Pavlyuk N., Borisov G., 2012, Peremennye Zvezdy, 32

Volnova A. A., et al., 2017, MNRAS, 467, 3500

Wang X., 2017, The Astronomer's Telegram, 10684, 1

Woosley S. E., Eastman R. G., Weaver T. A., Pinto P. A., 1994, ApJ, 429, 300

Woosley S. E., Kasen D., Blinnikov S., Sorokina E., 2007, ApJ, 662, 487

Yoon S.-C., Dessart L., Clocchiatti A., 2017, ApJ, 840, 10

van der Walt S., Colbert S. C., Varoquaux G., 2011, Computing in Science and Engineering, 13, 22

\section{APPENDIX A: TABLE}




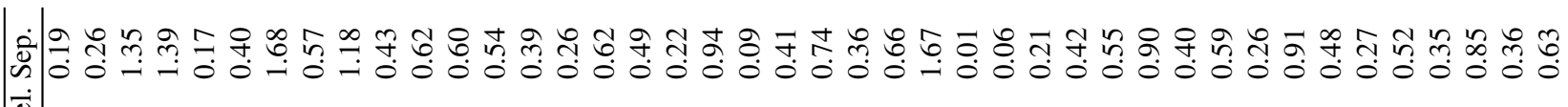
$\stackrel{\vec{\Phi}}{\mathscr{2}}$

ত্ำ 西

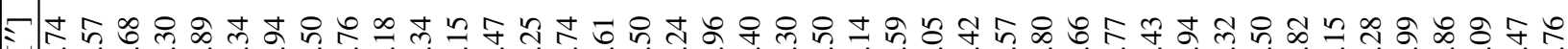

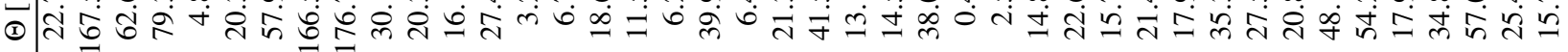

इ⿻ 의 m 行

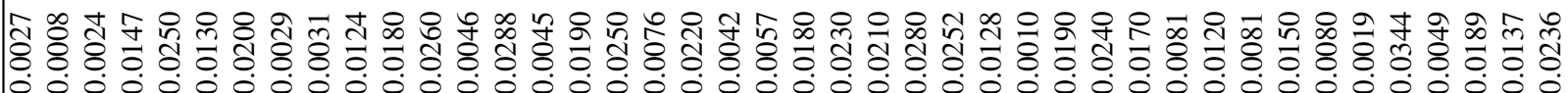

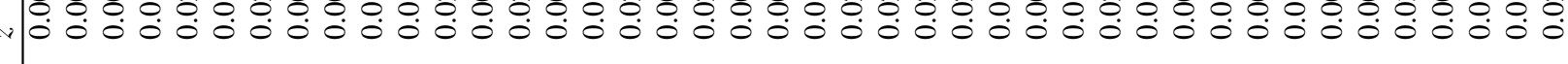

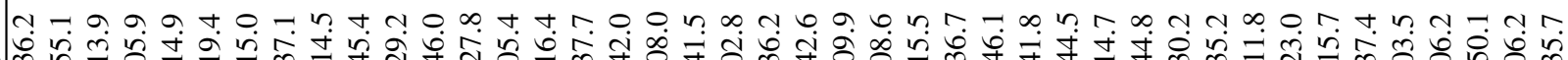

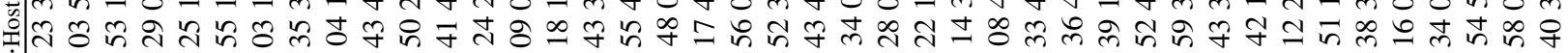

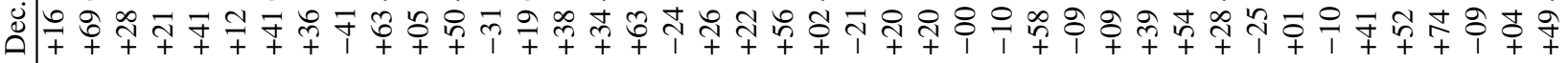

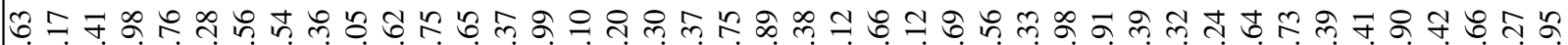

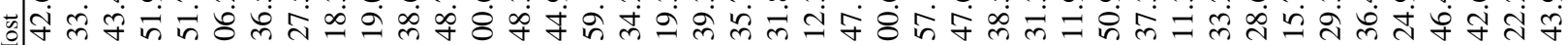

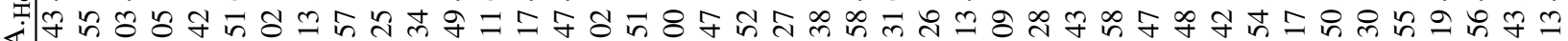
뜽

ant

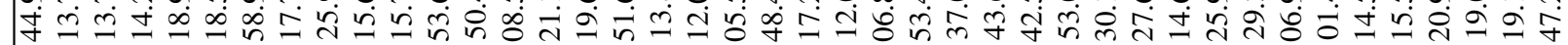
ఈ

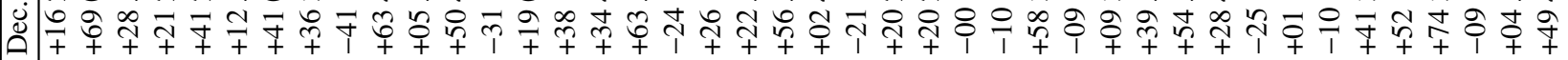

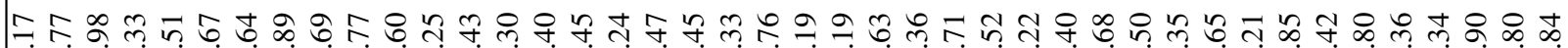

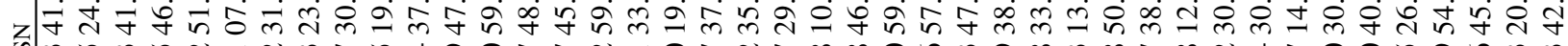

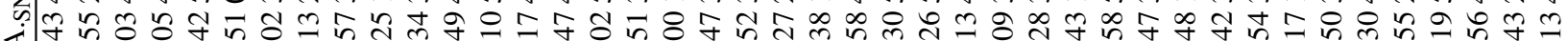

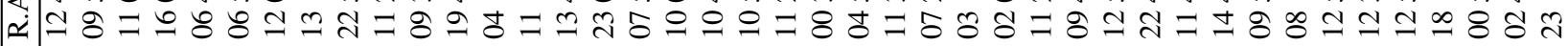

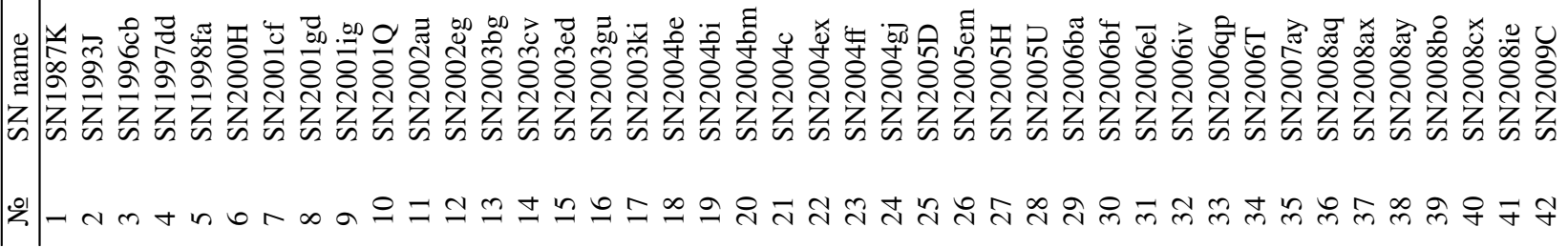




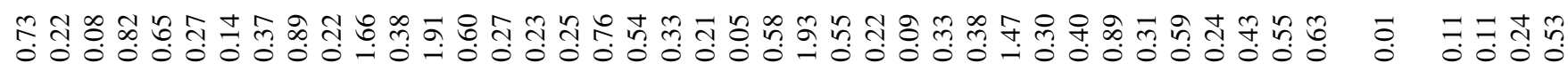

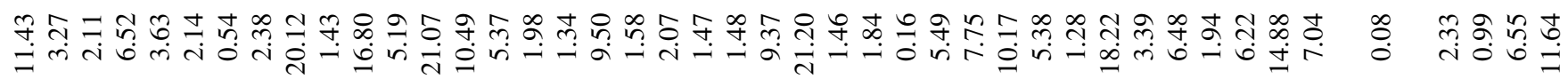

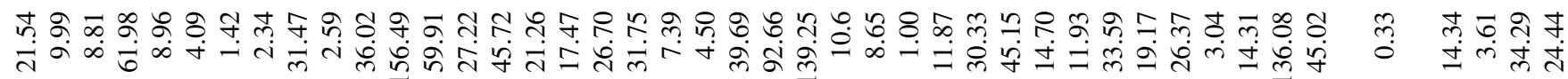

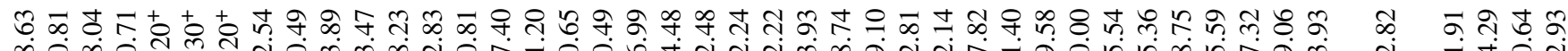

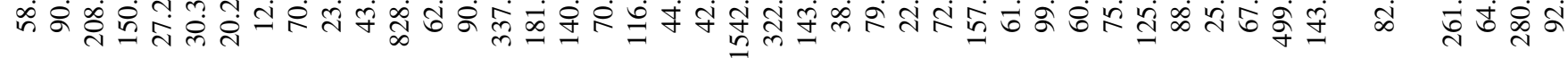

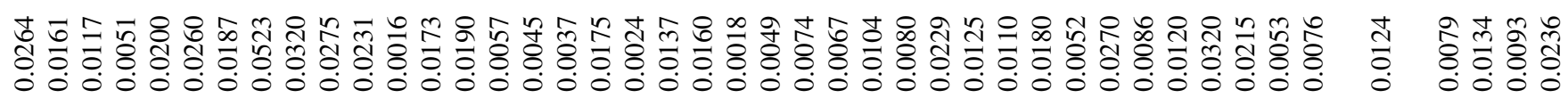

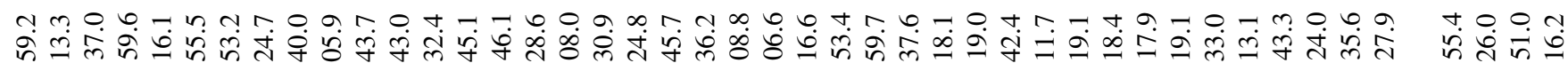

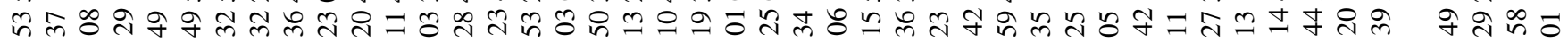

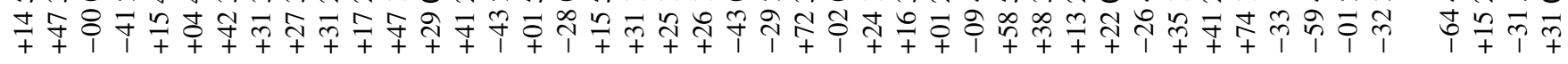

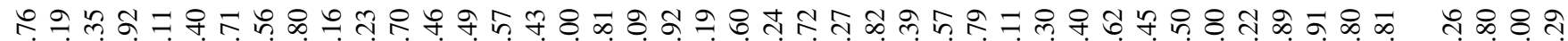

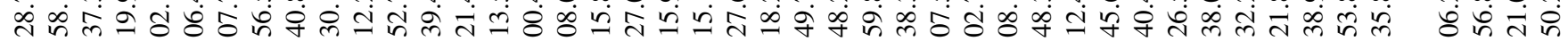

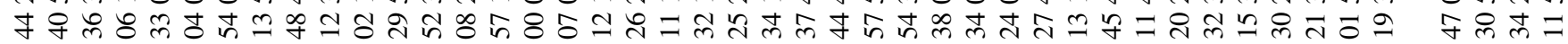

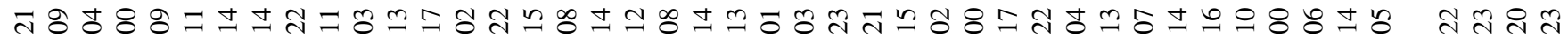

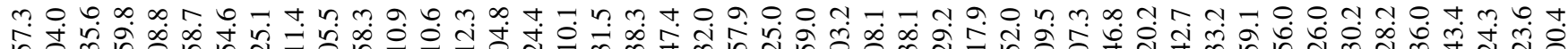
nूm

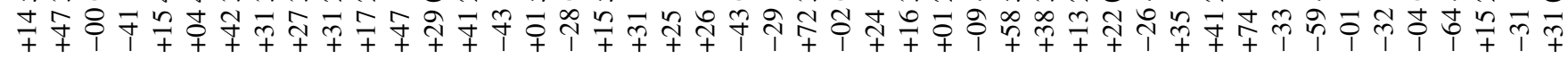

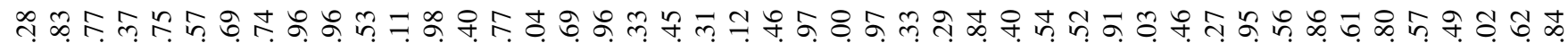

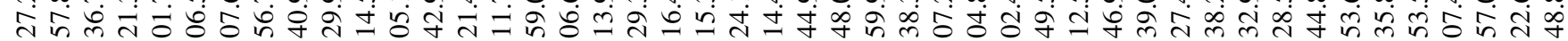

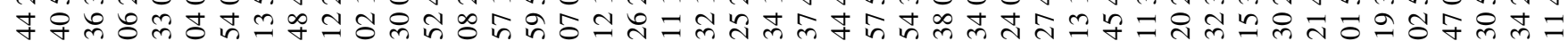
ส่

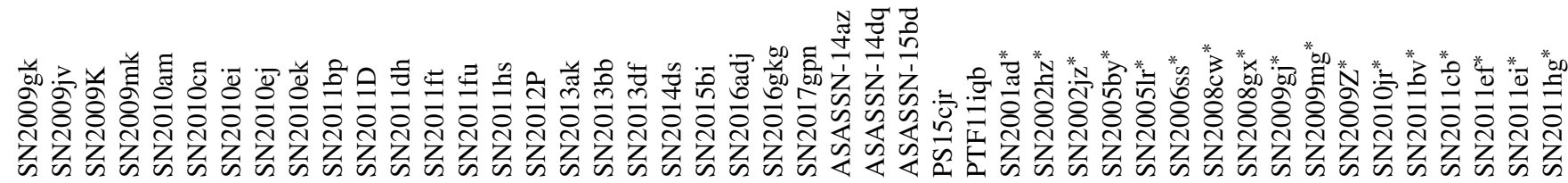

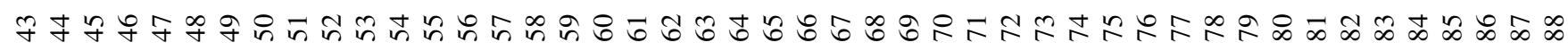




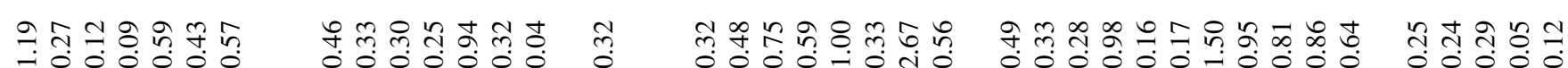

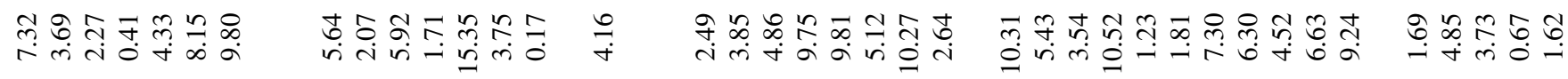

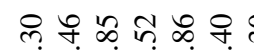

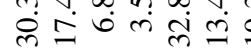

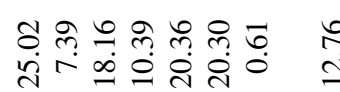

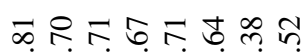

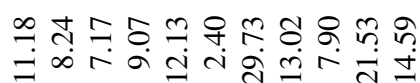

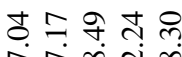

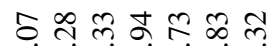

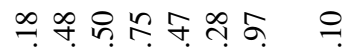

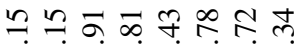

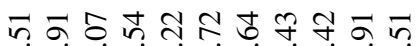

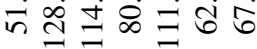

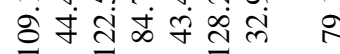
ठं

లి in in i⿱

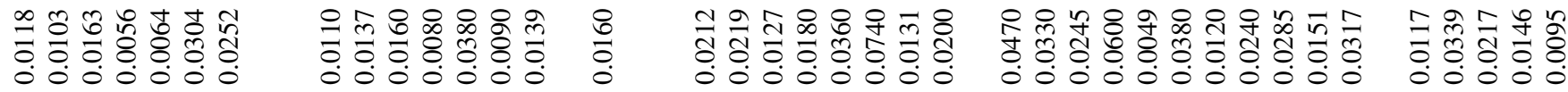

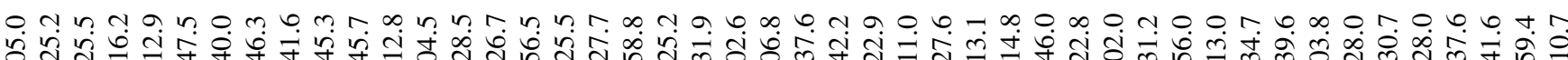

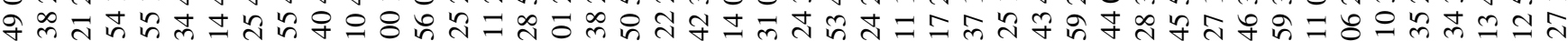

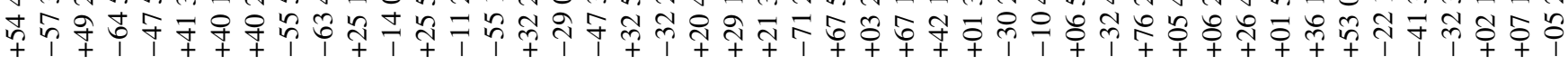

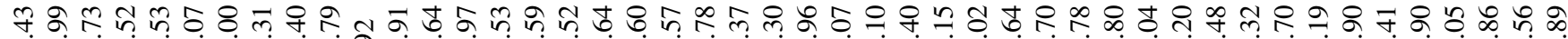

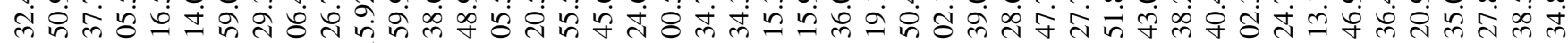

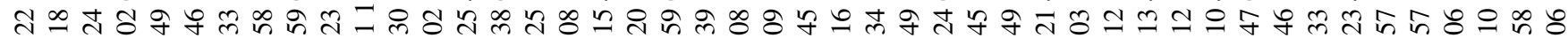
m

o.n

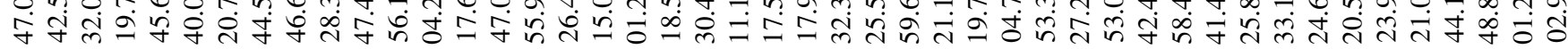

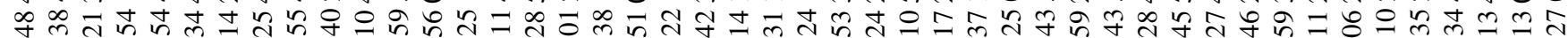

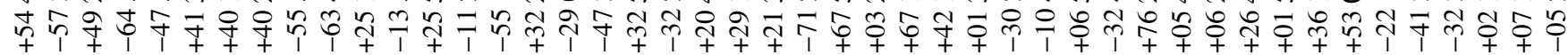

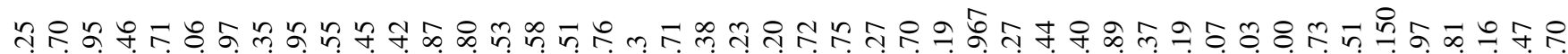

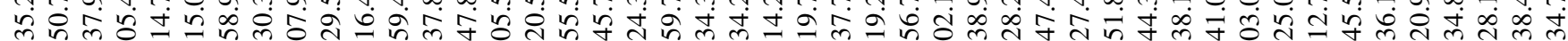

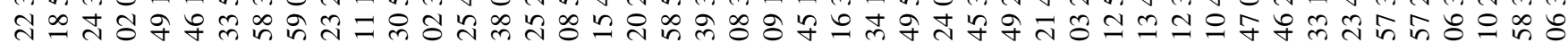
m

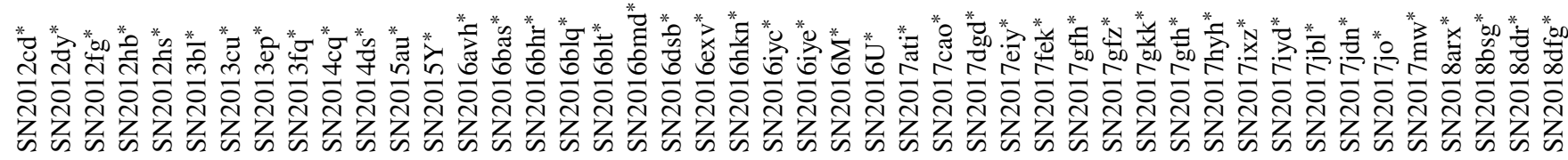

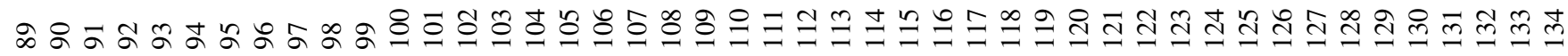




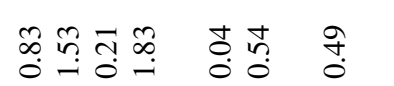

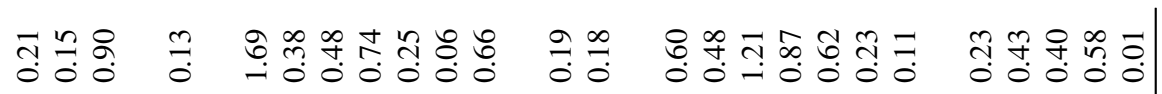

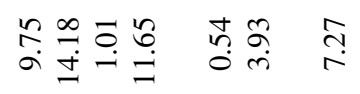

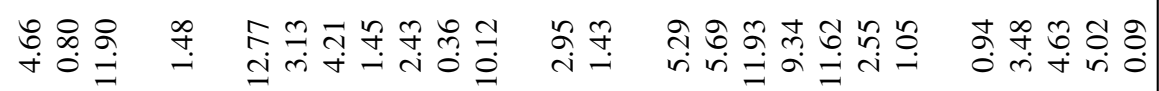

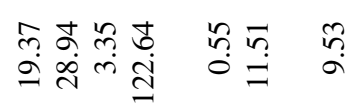

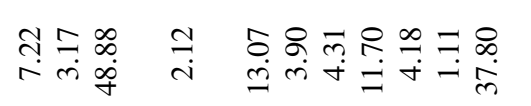

ले तु

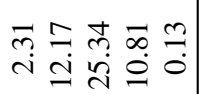

का

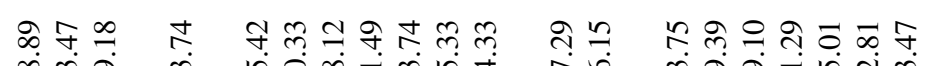

s. $8.8 \%$

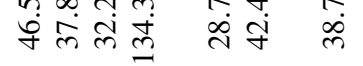

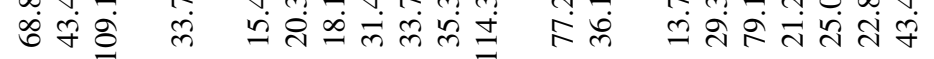

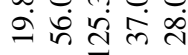

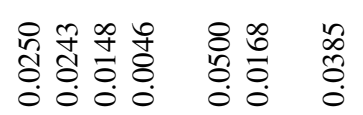

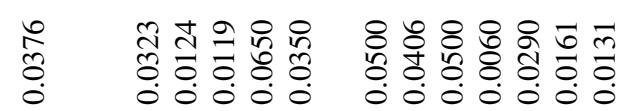

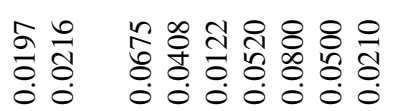

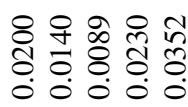

它

$\infty$ \&

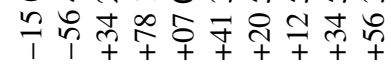

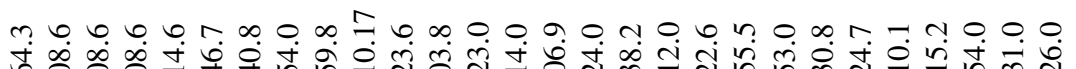

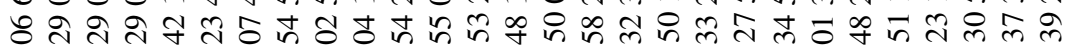

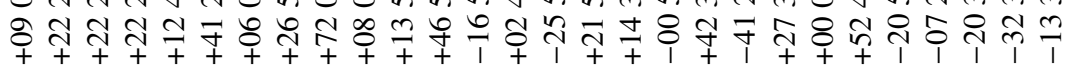

$\underset{0}{0} 0$.

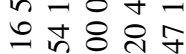

iิ

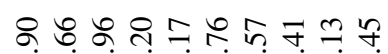
约 ㄴำ

ஓปন兀

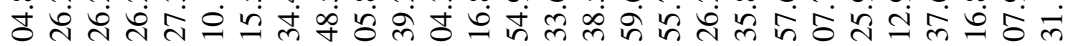

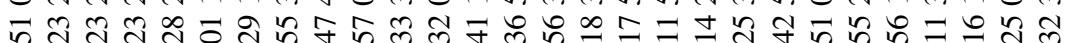
प

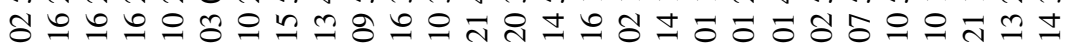

\& ชं

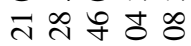
กำ

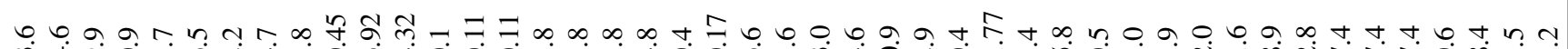
守吉 舟讨

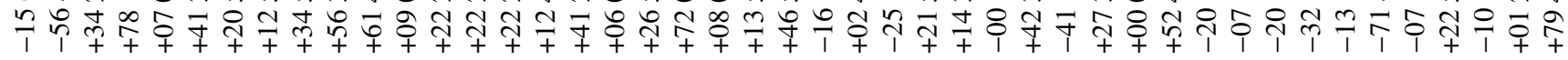

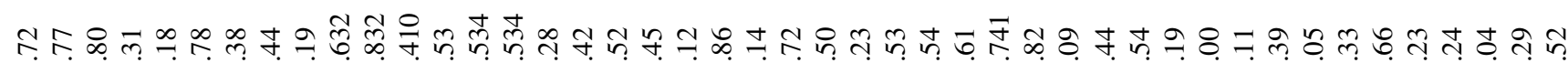

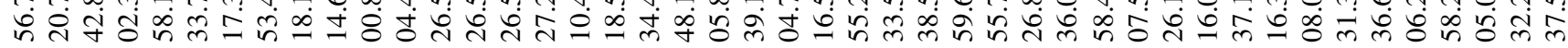
ไท

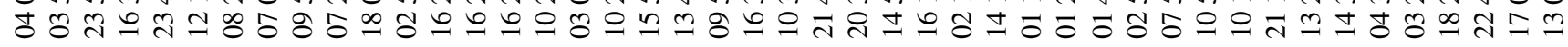

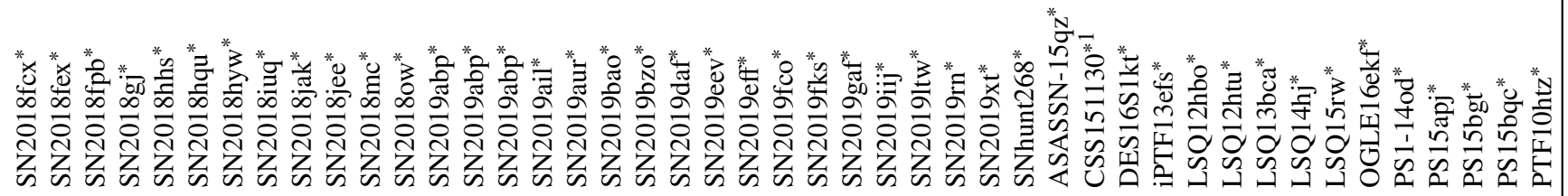

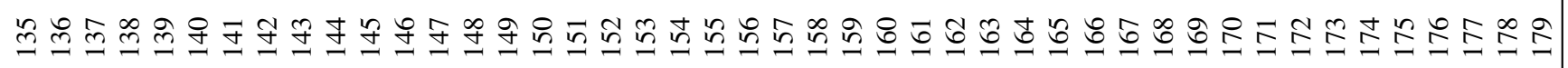




\section{E. A. Balakina et al.}

This paper has been typeset from a $\mathrm{T}_{\mathrm{E}} \mathrm{X} / \mathrm{LAT}_{\mathrm{E}} \mathrm{X}$ file prepared by the author. 